\title{
Rhodium-Catalyzed Multi-Component-Coupling Reactions Involving a Carborhodation-Cross-Coupling Sequence
}

\author{
Ryo Shintani, * Takafumi Yamagami, and Tamio Hayashi* \\ Department of Chemistry, Graduate School of Science, Kyoto University, \\ Sakyo, Kyoto 606-8502, Japan
}

\section{Supporting Information}

\section{General}

All air- and moisture-sensitive manipulations were carried out with standard Schlenk techniques under nitrogen or in a glove box under argon.

THF and toluene were purified by passing through a neutral alumina column under nitrogen. Benzene and 1,4-dioxane were distilled over benzophenone ketyl under nitrogen. Dichloromethane was distilled over $\mathrm{CaH}_{2}$ under nitrogen. Triethylamine was distilled over $\mathrm{KOH}$ under vacuum.

1-Hexyne (Wako Chemicals), phenylacetylene (Wako Chemicals), 4-methyl-1pentyne (TCI), 2-iodoaniline (Wako Chemicals), 2,4-diiodoaniline (Wako Chemicals), 4-iodoaniline (Wako Chemicals), iodomethane (Wako Chemicals), 3-bromo-2methyl-1-propene (Aldrich), pyridine (Wako Chemicals), acetic anhydride (Wako Chemicals), phenyl isocyanate (Wako Chemicals), diphenylphosphoryl azide (TCI), $N, N^{\prime}$-dicyclohexylcarbodiimide (Wako Chemicals), 2-iodobenzoic acid (Wako Chemicals), $\mathrm{ZnCl}_{2}$ (Wako Chemicals), $\mathrm{NaH}$ (Kanto Chemicals; $60 \mathrm{wt} \%$ in mineral oil), $t$-BuLi (Kanto Chemicals; $1.46 \mathrm{M}$ solution in pentane), MeLi (Kanto Chemicals; 1.04 $\mathrm{M}$ solution in $\mathrm{Et}_{2} \mathrm{O}$ ), 1,3-bis(diphenylphosphino)propane (Kanto Chemicals), 1,4bis(diphenylphosphino)butane (TCI), and 1,1'-bis(diphenylphosphino)ferrocene (Fluka) were used as received.

5-Methyl-2-hexynoic acid, 4-methyl-2-pentynoic acid, and 2-heptynoic acid were prepared from the corresponding terminal alkyne following the literature procedure. ${ }^{1}$ 5-Methoxy-1-pentyne, ${ }^{2}[\mathrm{RhCl}(\mathrm{cod})]_{2}{ }^{3}\left[\mathrm{RhCl}\left(\mathrm{C}_{2} \mathrm{H}_{4}\right)_{2}\right]_{2},{ }^{4}$ and $\mathrm{Pd}\left(\mathrm{PPh}_{3}\right)_{4}{ }^{5}$ were were synthesized following the literature procedures.

All other chemicals and solvents were purchased from Aldrich, Wako Chemicals, TCI, or Kanto Chemicals and used as received.

\footnotetext{
1 Tsou, H.-R.; Mamuya, N.; Johnson, B. D.; Reich, M. F.; Gruber, B. C.; Ye, F.; Nilakantan, R.; Shen, R.; Discafani, C.; DeBlanc, R.; Davis, R.; Koehn, F. E.; Greenberger, L. M.; Wang, Y.-F.; Wissner, A. J. Med. Chem. 2001, 44, 2719.

${ }^{2}$ Jackson, W. R.; Perlmutter, P.; Smallridge, A. J. Australian J. Chem. 1988, 41, 251.

${ }^{3}$ Giordano, G.; Crabtree, R. H. Inorg. Synth. 1979, 19, 218.

${ }^{4}$ Cramer, R. Inorg. Synth. 1974, 15, 16.

${ }^{5}$ Coulson, D. R. Inorg. Synth. 1972, 13, 121.
} 


\section{Synthesis of Substrates}

The yields have not been optimized.

\section{$\mathrm{N}$-(2-Iodophenyl)- $\mathrm{N}$-methyl-2-heptynamide (1a)}

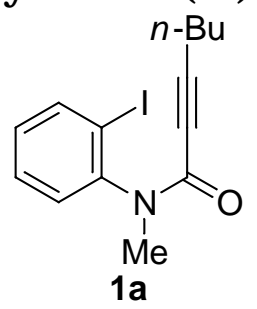

Diphenylphosphoryl azide $(5.50 \mathrm{~g}, 20.0 \mathrm{mmol})$ was added to a solution of 2iodobenzoic acid $(4.96 \mathrm{~g}, 20.0 \mathrm{mmol})$ and triethylamine $(2.02 \mathrm{~g}, 20.0 \mathrm{mmol})$ in benzene $(20 \mathrm{~mL})$ at $0{ }^{\circ} \mathrm{C}$. After stirring for $2 \mathrm{~h}$ at $0{ }^{\circ} \mathrm{C}$, the reaction mixture was washed with water and $\mathrm{NaCl}$ (saturated, aqueous), dried over $\mathrm{MgSO}_{4}$, and filtered. The resulting solution was refluxed for $2 \mathrm{~h}$ and the volatiles were removed under vacuum. The residue was dissolved in benzene $(20 \mathrm{~mL})$ and was then added to a solution of 1-hexynylmagnesium bromide $(20.0 \mathrm{mmol})$ in THF $(8.0 \mathrm{~mL})$ [prepared from ethylmagnesium bromide $(20.0 \mathrm{mmol})$ and 1-hexyne $(1.64 \mathrm{~g}, 20.0 \mathrm{mmol})$ at 0 $\left.{ }^{\circ} \mathrm{C}\right]$. The reaction mixture was stirred for $12 \mathrm{~h}$ at room temperature and quenched with $\mathrm{NH}_{4} \mathrm{Cl}$ (saturated, aqueous). After extraction with $\mathrm{Et}_{2} \mathrm{O}$, the organic layer was washed successively with $\mathrm{HCl}\left(10 \%\right.$ aqueous), $\mathrm{NaHCO}_{3}$ (saturated aqueous), and $\mathrm{NaCl}$ (aqueous), and dried over $\mathrm{MgSO}_{4}$, filtered, and concentrated under vacuum. The residue was chromatographed on silica gel with $\mathrm{CH}_{2} \mathrm{Cl}_{2} /$ hexane $=2 / 1$ to afford $\mathrm{N}$-(2-iodophenyl)-2-heptynamide as a pale yellow solid (1.79 $\mathrm{g}, 5.47 \mathrm{mmol}$ ).

A solution of this amide in THF $(15 \mathrm{~mL})$ was added to a suspension of $\mathrm{NaH}(240$ $\mathrm{mg}, 6.00 \mathrm{mmol} ; 60 \mathrm{wt}^{\circ}$ in mineral oil) in THF $(5 \mathrm{~mL})$ at $0{ }^{\circ} \mathrm{C}$, and the resulting mixture was stirred for $1 \mathrm{~h}$ at $0{ }^{\circ} \mathrm{C}$. Iodomethane $(851 \mathrm{mg}, 6.00 \mathrm{mmol})$ was then added to it dropwise, and the mixture was stirred for $16 \mathrm{~h}$ at room temperature. The reaction was quenched with water and extracted with $\mathrm{Et}_{2} \mathrm{O}$. The organic layer was washed with $\mathrm{NaCl}$ (saturated, aqueous), dried over $\mathrm{MgSO}_{4}$, filtered, and concentrated under vacuum. The residue was chromatographed on silica gel with $\mathrm{Et}_{2} \mathrm{O} /$ hexane $=1 / 1$ to afford compound 1a as a pale yellow oil $(660 \mathrm{mg}, 1.93 \mathrm{mmol}$; $10 \%$ overall yield). This compound exists as a 10:1 mixture of rotamers.

Major rotamer: ${ }^{1} \mathrm{H}$ NMR $\left(\mathrm{CDCl}_{3}\right): \delta 7.91\left(\mathrm{dd},{ }^{3} J_{\mathrm{HH}}=7.9 \mathrm{~Hz}\right.$ and $\left.{ }^{4} J_{\mathrm{HH}}=1.3 \mathrm{~Hz}, 1 \mathrm{H}\right)$, $7.42\left(\mathrm{ddd},{ }^{3} J_{\mathrm{HH}}=7.8\right.$ and $7.4 \mathrm{~Hz}$ and $\left.{ }^{4} \mathrm{~J}_{\mathrm{HH}}=1.3 \mathrm{~Hz}, 1 \mathrm{H}\right), 7.31\left(\mathrm{dd},{ }^{3} J_{\mathrm{HH}}=7.8 \mathrm{~Hz}\right.$ and $\left.{ }^{4} J_{\mathrm{HH}}=1.6 \mathrm{~Hz}, 1 \mathrm{H}\right), 7.09\left(\mathrm{ddd},{ }^{3} J_{\mathrm{HH}}=7.8\right.$ and $7.4 \mathrm{~Hz}$ and $\left.{ }^{4} J_{\mathrm{HH}}=1.6 \mathrm{~Hz}, 1 \mathrm{H}\right), 3.22(\mathrm{~s}$, $3 \mathrm{H}), 2.11-2.01(\mathrm{~m}, 2 \mathrm{H}), 1.20-1.14(\mathrm{~m}, 2 \mathrm{H}), 1.07-0.98(\mathrm{~m}, 2 \mathrm{H}), 0.73\left(\mathrm{t},{ }^{3} \mathrm{~J}_{\mathrm{HH}}=7.2 \mathrm{~Hz}, 3 \mathrm{H}\right)$. ${ }^{13} \mathrm{C}$ NMR $\left(\mathrm{CDCl}_{3}\right): \delta 154.2,145.5,139.6,129.7,129.4,129.3,99.5,94.3,74.7,35.0,29.2$, 21.2, 18.1, 13.3. Anal. Calcd for $\mathrm{C}_{14} \mathrm{H}_{16} \mathrm{INO}: \mathrm{C}, 49.28 ; \mathrm{H}, 4.73$. Found: $\mathrm{C}, 49.03 ; \mathrm{H}$, 4.51 .

\section{$N$-(2-Iodophenyl)- $N$-methyl-6-methoxy-2-hexynamide (1b)}<smiles>COCCCC#CC(=O)N(C)c1ccccc1I</smiles> 
This was synthesized from 2-iodobenzoic acid and 5-methoxy-1-pentyne, following the procedure for compound 1a. Brown oil, $26 \%$ overall yield. This compound exists as a 9.5:1 mixture of rotamers.

Major rotamer: ${ }^{1} \mathrm{H}$ NMR $\left(\mathrm{CDCl}_{3}\right): \delta 7.92\left(\mathrm{dd},{ }^{3} J_{\mathrm{HH}}=7.9 \mathrm{~Hz}\right.$ and $\left.{ }^{4} J_{\mathrm{HH}}=1.2 \mathrm{~Hz}, 1 \mathrm{H}\right)$, $7.43\left(\mathrm{td},{ }^{3} J_{\mathrm{HH}}=7.6 \mathrm{~Hz}\right.$ and $\left.{ }^{4} J_{\mathrm{HH}}=1.3 \mathrm{~Hz}, 1 \mathrm{H}\right), 7.32\left(\mathrm{dd},{ }^{3} J_{\mathrm{HH}}=7.8 \mathrm{~Hz}\right.$ and ${ }^{4} J_{\mathrm{HH}}=1.5$ $\mathrm{Hz}, 1 \mathrm{H}), 7.09\left(\mathrm{td},{ }^{3} J_{\mathrm{HH}}=7.7 \mathrm{~Hz},{ }^{4} J_{\mathrm{HH}}=1.5 \mathrm{~Hz}, 1 \mathrm{H}\right), 3.24(\mathrm{~s}, 3 \mathrm{H}), 3.22(\mathrm{~s}, 3 \mathrm{H}), 3.12-3.07$ $(\mathrm{m}, 2 \mathrm{H}), 2.21-2.10(\mathrm{~m}, 2 \mathrm{H}), 1.50-1.38(\mathrm{~m}, 2 \mathrm{H}) .{ }^{13} \mathrm{C}$ NMR $\left(\mathrm{CDCl}_{3}\right): \delta 154.2,145.6,140.0$, 129.9, 129.5, 129.4, 99.6, 92.9, 74.9, 70.4, 58.5, 35.1, 27.6, 15.3. Anal. Calcd for $\mathrm{C}_{14} \mathrm{H}_{16} \mathrm{INO}_{2}$ : C, $47.08 ; \mathrm{H}, 4.52$. Found: $\mathrm{C}, 47.07 ; \mathrm{H}, 4.45$.

\section{$N$-(2-Iodophenyl)-N,5-dimethyl-2-hexynamide (1c)}<smiles>CN(C(=O)C#CC(C)(C)C)c1ccccc1I</smiles>

A solution of DCC $(1.90 \mathrm{~g}, 9.21 \mathrm{mmol})$ in $\mathrm{CH}_{2} \mathrm{Cl}_{2}(10 \mathrm{~mL})$ was added to a solution of 2-iodoaniline (2.01 g, $9.18 \mathrm{mmol})$ and 5-methyl-2-hexynoic acid $(1.16 \mathrm{~g}, 9.20 \mathrm{mmol})$ in $\mathrm{CH}_{2} \mathrm{Cl}_{2}(10 \mathrm{~mL})$ at $0{ }^{\circ} \mathrm{C}$, and the mixture was stirred for $18 \mathrm{~h}$ at $0{ }^{\circ} \mathrm{C}$. The precipitate was filtered off through celite, and the solvent was removed under vacuum to afford $\mathrm{N}$-(2-iodophenyl)-5-methyl-2-hexynamide as a yellow solid (1.00 g, $3.06 \mathrm{mmol}$ ).

A solution of this amide in THF $(9 \mathrm{~mL})$ was added to a suspension of $\mathrm{NaH}(134$ $\mathrm{mg}, 3.35 \mathrm{mmol} ; 60 \mathrm{wt} \%$ in mineral oil) in THF $(3 \mathrm{~mL})$ at $0{ }^{\circ} \mathrm{C}$, and the resulting mixture was stirred for $1 \mathrm{~h}$ at $0{ }^{\circ} \mathrm{C}$. Iodomethane $(851 \mathrm{mg}, 3.36 \mathrm{mmol})$ was then added to it dropwise, and the mixture was stirred for $16 \mathrm{~h}$ at room temperature. The reaction was quenched with water and extracted with $\mathrm{Et}_{2} \mathrm{O}$. The organic layer was dried over $\mathrm{MgSO}_{4}$, filtered, and concentrated under vacuum. The residue was chromatographed on silica gel with $\mathrm{Et}_{2} \mathrm{O} /$ hexane $=3 / 2$ to afford compound $\mathbf{1 c}$ as a pale yellow solid (1.01 g, $2.96 \mathrm{mmol} ; 32 \%$ overall yield). This compound exists as an 11:1 mixture of rotamers.

Major rotamer: ${ }^{1} \mathrm{H}$ NMR $\left(\mathrm{CDCl}_{3}\right): \delta 7.91\left(\mathrm{~d},{ }^{3} J_{\mathrm{HH}}=7.9 \mathrm{~Hz}, 1 \mathrm{H}\right), 7.41\left(\mathrm{t},{ }^{3} J_{\mathrm{HH}}=7.6\right.$ $\mathrm{Hz}, 1 \mathrm{H}), 7.32\left(\mathrm{~d},{ }^{3} J_{\mathrm{HH}}=7.7 \mathrm{~Hz}, 1 \mathrm{H}\right), 7.07\left(\mathrm{t},{ }^{3} J_{\mathrm{HH}}=7.6 \mathrm{~Hz}, 1 \mathrm{H}\right), 3.22(\mathrm{~s}, 3 \mathrm{H}), 2.03-1.93$ $(\mathrm{m}, 2 \mathrm{H}), 1.55-1.47(\mathrm{~m}, 1 \mathrm{H}), 0.66\left(\mathrm{~d},{ }^{3} J_{\mathrm{HH}}=6.7 \mathrm{~Hz}, 3 \mathrm{H}\right), 0.64\left(\mathrm{~d},{ }^{3} J_{\mathrm{HH}}=6.7 \mathrm{~Hz}, 3 \mathrm{H}\right) .{ }^{13} \mathrm{C}$ NMR $\left(\mathrm{CDCl}_{3}\right): \delta 154.4,145.7,139.8,129.9,129.6,129.5,99.7,92.6,75.6,35.1,27.7,27.2$, 21.6, 21.5. Anal. Calcd for $\mathrm{C}_{14} \mathrm{H}_{16} \mathrm{INO}$ : C, 49.28; $\mathrm{H}, 4.73$. Found: $\mathrm{C}, 49.40 ; \mathrm{H}, 4.76$.

\section{$N$-(2-Iodophenyl)- $N$,4-dimethyl-2-pentynamide (1d)}<smiles>CCCI</smiles>

This was synthesized from 2-iodoaniline and 4-methyl-2-pentynoic acid, following the procedure for compound 1c. Pale yellow oil, 32\% overall yield. This compound exists as a 10:1 mixture of rotamers.

Major rotamer: ${ }^{1} \mathrm{H} \mathrm{NMR}\left(\mathrm{CDCl}_{3}\right): \delta 7.91\left(\mathrm{dd},{ }^{3} J_{\mathrm{HH}}=7.9 \mathrm{~Hz}\right.$ and $\left.{ }^{4} J_{\mathrm{HH}}=1.3 \mathrm{~Hz}, 1 \mathrm{H}\right)$, 
$7.41\left(\mathrm{td},{ }^{3} J_{\mathrm{HH}}=7.6 \mathrm{~Hz}\right.$ and $\left.{ }^{4} J_{\mathrm{HH}}=1.4 \mathrm{~Hz}, 1 \mathrm{H}\right), 7.30\left(\mathrm{dd},{ }^{3} J_{\mathrm{HH}}=7.8 \mathrm{~Hz}\right.$ and ${ }^{4} J_{\mathrm{HH}}=1.6$ $\mathrm{Hz}, 1 \mathrm{H}), 7.09\left(\mathrm{ddd},{ }^{3} \mathrm{~J}_{\mathrm{HH}}=7.7\right.$ and $7.4 \mathrm{~Hz}$ and $\left.{ }^{4} J_{\mathrm{HH}}=1.6 \mathrm{~Hz}, 1 \mathrm{H}\right), 3.22(\mathrm{~s}, 3 \mathrm{H}), 2.37$ (heptet, $\left.{ }^{3} \mathrm{~J}_{\mathrm{HH}}=6.8 \mathrm{~Hz}, 1 \mathrm{H}\right), 0.88\left(\mathrm{~d},{ }^{3} \mathrm{~J}_{\mathrm{HH}}=6.8 \mathrm{~Hz}, 3 \mathrm{H}\right), 0.85\left(\mathrm{~d},{ }^{3} \mathrm{~J}_{\mathrm{HH}}=6.8 \mathrm{~Hz}, 3 \mathrm{H}\right) .{ }^{13} \mathrm{C}$ NMR $\left(\mathrm{CDCl}_{3}\right): \delta 154.2,145.5,139.4,129.6,129.3,129.2,99.5,98.1,73.8,34.8,21.3,21.3$, 20.0. Anal. Calcd for $\mathrm{C}_{13} \mathrm{H}_{14} \mathrm{INO}$ : $\mathrm{C}, 47.73 ; \mathrm{H}, 4.31$. Found: $\mathrm{C}, 47.74 ; \mathrm{H}, 4.24$.

\section{$N$-(2-Iodophenyl)- $N$-methyl-3-phenylpropynamide (1e)}<smiles>CN(C(=O)C#Cc1ccccc1)c1ccccc1I</smiles>

This was synthesized from 2-iodobenzoic acid and phenylacetylene, following the procedure for compound 1a. Orange solid, 10\% overall yield. This compound exists as an 11:1 mixture of rotamers.

Major rotamer: ${ }^{1} \mathrm{H}$ NMR $\left(\mathrm{CDCl}_{3}\right): \delta 7.97\left(\mathrm{~d},{ }^{3} \mathrm{~J}_{\mathrm{HH}}=7.9 \mathrm{~Hz}, 1 \mathrm{H}\right), 7.46\left(\mathrm{t},{ }^{3} J_{\mathrm{HH}}=7.6\right.$ $\mathrm{Hz}, 1 \mathrm{H}), 7.39\left(\mathrm{dd},{ }^{3} J_{\mathrm{HH}}=7.7 \mathrm{~Hz}\right.$ and $\left.{ }^{4} J_{\mathrm{HH}}=1.3 \mathrm{~Hz}, 1 \mathrm{H}\right), 7.32\left(\mathrm{t},{ }^{3} J_{\mathrm{HH}}=7.4 \mathrm{~Hz}, 1 \mathrm{H}\right)$, $7.22\left(\mathrm{t},{ }^{3} J_{\mathrm{HH}}=7.7 \mathrm{~Hz}, 2 \mathrm{H}\right), 7.14\left(\mathrm{td},{ }^{3} J_{\mathrm{HH}}=7.7 \mathrm{~Hz}\right.$ and $\left.{ }^{4} J_{\mathrm{HH}}=1.3 \mathrm{~Hz}, 1 \mathrm{H}\right), 7.08\left(\mathrm{~d},{ }^{3} J_{\mathrm{HH}}\right.$ $=7.4 \mathrm{~Hz}, 2 \mathrm{H}), 3.30(\mathrm{~s}, 3 \mathrm{H}) .{ }^{13} \mathrm{C} \mathrm{NMR}\left(\mathrm{CDCl}_{3}\right): \delta 154.3,145.5,139.8,132.5,130.1,130.0$, 129.7, 129.5, 128.3, 120.2, 99.7, 90.6, 82.3, 35.2. Anal. Calcd for $\mathrm{C}_{16} \mathrm{H}_{12} \mathrm{INO}$ : C, 53.21; H, 3.35. Found: C, 53.24; H, 3.45.

\section{$N$-(2-Iodophenyl)- $N$-methylacetamide (3)}<smiles>CC(=O)N(C)c1ccccc1I</smiles>

Pyridine $(0.61 \mathrm{~mL}, 7.54 \mathrm{mmol})$ and acetic anhydride $(0.71 \mathrm{~mL}, 7.51 \mathrm{mmol})$ were successively added to a solution of 2-iodoaniline $(1.10 \mathrm{~g}, 5.02 \mathrm{mmol})$ in $\mathrm{CH}_{2} \mathrm{Cl}_{2}(10$ $\mathrm{mL}$ ) at $0{ }^{\circ} \mathrm{C}$, and the mixture was stirred for $12 \mathrm{~h}$ at room temperature. The reaction was quenched with water and extracted with $\mathrm{CH}_{2} \mathrm{Cl}_{2}$. The organic layer was washed with $\mathrm{NaCl}$ (saturated, aqueous), dried over $\mathrm{MgSO}_{4}$, filtered, and concentrated under vacuum to afford $N$-(2-iodophenyl)acetamide as a pale orange solid (1.02 $\mathrm{g}, 3.91$ mmol).

A solution of this amide in THF (10 mL) was added to a suspension of $\mathrm{NaH}(175$ $\mathrm{mg}, 4.38 \mathrm{mmol} ; 60 \mathrm{wt} \%$ in mineral oil $)$ in THF $(4 \mathrm{~mL})$ at $0{ }^{\circ} \mathrm{C}$, and the resulting mixture was stirred for $1 \mathrm{~h}$ at $0{ }^{\circ} \mathrm{C}$. Iodomethane $(625 \mathrm{mg}, 4.40 \mathrm{mmol})$ was then added to it dropwise, and the mixture was stirred for $21 \mathrm{~h}$ at room temperature. The reaction was quenched with water and extracted with $\mathrm{Et}_{2} \mathrm{O}$. The organic layer was dried over $\mathrm{MgSO}_{4}$, filtered, and concentrated under vacuum. The residue was chromatographed on silica gel with $\mathrm{Et}_{2} \mathrm{O} /$ hexane $=1 / 1$ to afford compound 3 as a white solid (680 mg, $2.74 \mathrm{mmol} ; 49 \%$ overall yield). This compound exists as a 33:1 mixture of rotamers.

Major rotamer: ${ }^{1} \mathrm{H} \mathrm{NMR}\left(\mathrm{CDCl}_{3}\right): \delta 7.94\left(\mathrm{dd},{ }^{3} J_{\mathrm{HH}}=7.9 \mathrm{~Hz}\right.$ and $\left.{ }^{4} J_{\mathrm{HH}}=1.4 \mathrm{~Hz}, 1 \mathrm{H}\right)$, $7.43\left(\mathrm{td},{ }^{3} J_{\mathrm{HH}}=7.6 \mathrm{~Hz}\right.$ and $\left.{ }^{4} J_{\mathrm{HH}}=1.4 \mathrm{~Hz}, 1 \mathrm{H}\right), 7.28\left(\mathrm{dd},{ }^{3} J_{\mathrm{HH}}=7.8 \mathrm{~Hz}\right.$ and ${ }^{4} J_{\mathrm{HH}}=1.6$ $\mathrm{Hz}, 1 \mathrm{H}), 7.08\left(\mathrm{td},{ }^{3} \mathrm{~J}_{\mathrm{HH}}=7.7 \mathrm{~Hz},{ }^{4} J_{\mathrm{HH}}=1.5 \mathrm{~Hz}, 1 \mathrm{H}\right), 3.18(\mathrm{~s}, 3 \mathrm{H}), 1.80(\mathrm{~s}, 3 \mathrm{H}) .{ }^{13} \mathrm{C} \mathrm{NMR}$ $\left(\mathrm{CDCl}_{3}\right): \delta 170.1,146.6,140.2,130.0,129.8,128.8,99.4,35.7,22.5$. Anal. Calcd for 
$\mathrm{C}_{9} \mathrm{H}_{10} \mathrm{INO}: \mathrm{C}, 39.30 ; \mathrm{H}, 3.66$. Found: C, 39.03; H, 3.64.

$N$-(4-Iodophenyl)- $N$-methyl-2-heptynamide (5)<smiles>CN(C(=O)C#CBr)c1ccc(Br)cc1</smiles>

This was synthesized from 4-iodoaniline and 2-heptynoic acid, following the procedure for compound 1c. White solid, 36\% overall yield. This compound exists as a 5.9:1 mixture of rotamers.

Major rotamer: ${ }^{1} \mathrm{H}$ NMR $\left(\mathrm{CDCl}_{3}\right): \delta 7.72\left(\mathrm{~d},{ }^{3} \mathrm{~J}_{\mathrm{HH}}=8.5 \mathrm{~Hz}, 2 \mathrm{H}\right), 7.03\left(\mathrm{~d},{ }^{3} \mathrm{~J}_{\mathrm{HH}}=8.4\right.$ $\mathrm{Hz}, 2 \mathrm{H}), 3.29(\mathrm{~s}, 3 \mathrm{H}), 2.13\left(\mathrm{t},{ }^{3} \mathrm{~J}_{\mathrm{HH}}=6.9 \mathrm{~Hz}, 2 \mathrm{H}\right), 1.27$ (quint, $\left.{ }^{3} J_{\mathrm{HH}}=7.2 \mathrm{~Hz}, 2 \mathrm{H}\right), 1.10$ (sextet, $\left.{ }^{3} J_{\mathrm{HH}}=7.4 \mathrm{~Hz}, 2 \mathrm{H}\right), 0.80\left(\mathrm{t},{ }^{3} \mathrm{~J}_{\mathrm{HH}}=7.3 \mathrm{~Hz}, 3 \mathrm{H}\right) .{ }^{13} \mathrm{C} \mathrm{NMR}\left(\mathrm{CDCl}_{3}\right): \delta 154.0$, 143.1, 138.2, 129.2, 94.5, 92.6, 74.8, 36.1, 29.3, 21.5, 18.4, 13.5. Anal. Calcd for $\mathrm{C}_{14} \mathrm{H}_{16} \mathrm{INO}: \mathrm{C}, 49.28 ; \mathrm{H}, 4.73$. Found: $\mathrm{C}, 49.22 ; \mathrm{H}, 4.65$.

\section{$N$-(2,4-Diiodophenyl)- $N$-methyl-2-heptynamide (8)}<smiles>CN(C(=O)C#CBr)c1ccc(I)cc1I</smiles>

This was synthesized from 2,4-diiodoaniline and 2-heptynoic acid, following the procedure for compound 1c. White solid, $8 \%$ overall yield. This compound exists as a 10:1 mixture of rotamers.

Major rotamer: ${ }^{1} \mathrm{H}$ NMR $\left(\mathrm{CDCl}_{3}\right): \delta 8.26\left(\mathrm{~d},{ }^{4} J_{\mathrm{HH}}=1.7,1 \mathrm{H}\right), 7.72\left(\mathrm{dd},{ }^{3} J_{\mathrm{HH}}=8.2 \mathrm{~Hz}\right.$ and $\left.{ }^{4} J_{\mathrm{HH}}=1.7 \mathrm{~Hz}, 1 \mathrm{H}\right), 7.03\left(\mathrm{~d},{ }^{3} J_{\mathrm{HH}}=8.3 \mathrm{~Hz}, 1 \mathrm{H}\right), 3.19(\mathrm{~s}, 3 \mathrm{H}), 2.17-2.06(\mathrm{~m}, 2 \mathrm{H}), 1.26-$ $1.17(\mathrm{~m}, 2 \mathrm{H}), 1.11-1.02(\mathrm{~m}, 2 \mathrm{H}), 0.80\left(\mathrm{t},{ }^{3} \mathrm{JHH}_{\mathrm{HH}}=7.3 \mathrm{~Hz}, 3 \mathrm{H}\right) .{ }^{13} \mathrm{C} \mathrm{NMR}\left(\mathrm{CDCl}_{3}\right): \delta 154.0$, 147.3, 145.4, 138.6, 130.9, 101.0, 94.2, 94.2, 74.7, 35.0, 29.3, 21.4, 18.3, 13.5. Anal. Calcd for $\mathrm{C}_{14} \mathrm{H}_{15} \mathrm{I}_{2} \mathrm{NO}$ : C, 36.00; H, 3.24. Found: $\mathrm{C}, 35.93 ; \mathrm{H}, 3.21$.

\section{$N$-(2-Methyl-2-propenyl)- $N$-phenyl-2-heptynamide (11a)}

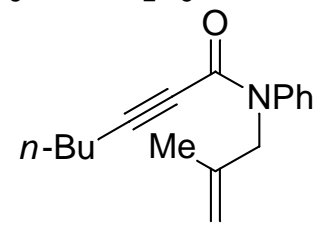

11a

A solution of phenyl isocyanate $(1.19 \mathrm{~g}, 10.0 \mathrm{mmol})$ in benzene $(10 \mathrm{~mL})$ was added to a solution of 1-hexynylmagnesium bromide $(10.0 \mathrm{mmol})$ in THF $(8.0 \mathrm{~mL})$ [prepared from ethylmagnesium bromide $(10.0 \mathrm{mmol})$ and 1-hexyne $(822 \mathrm{mg}, 10.0$ $\mathrm{mmol}$ ) at $0{ }^{\circ} \mathrm{C}$ ]. The reaction mixture was stirred for $21 \mathrm{~h}$ at room temperature, quenched with $\mathrm{NH}_{4} \mathrm{Cl}$ (saturated, aqueous), and extracted with $\mathrm{Et}_{2} \mathrm{O}$. The organic layer was washed successively with $\mathrm{HCl}$ (10\% aqueous), $\mathrm{NaHCO}_{3}$ (saturated, aqueous), and $\mathrm{NaCl}$ (saturated, aqueous), and dried over $\mathrm{MgSO}_{4}$, filtered, and concentrated under vacuum. The residue was chromatographed on silica gel with 
$\mathrm{Et}_{2} \mathrm{O} /$ hexane $=2 / 1$ to afford $N$-phenyl-2-heptynamide as a pale yellow oil (1.65 $\mathrm{g}$, $8.20 \mathrm{mmol})$.

A solution of this amide $(1.65 \mathrm{~g}, 8.20 \mathrm{mmol})$ in THF $(10 \mathrm{~mL})$ was added to a suspension of $\mathrm{NaH}(361 \mathrm{mg}, 9.03 \mathrm{mmol} ; 60 \mathrm{wt} \%$ in mineral oil) in THF (10 mL) at 0 ${ }^{\circ} \mathrm{C}$, and the resulting mixture was stirred for $1 \mathrm{~h}$ at $0{ }^{\circ} \mathrm{C}$. 3-Bromo-2-methyl-1propene $(1.25 \mathrm{~g}, 9.22 \mathrm{mmol})$ was then added to it dropwise, and the mixture was stirred for $18 \mathrm{~h}$ at room temperature. The reaction was quenched with water and extracted with $\mathrm{Et}_{2} \mathrm{O}$. The organic layer was washed with $\mathrm{NaCl}$ (saturated, aqueous), dried over $\mathrm{MgSO}_{4}$, filtered, and concentrated under vacuum. The residue was chromatographed on silica gel with $\mathrm{Et}_{2} \mathrm{O} /$ hexane $=1 / 3$ to afford compound 11a as a yellow oil (1.50 g, $5.87 \mathrm{mmol} ; 59 \%$ overall yield). This compound exists as a 4.9:1 mixture of rotamers.

Major rotamer: ${ }^{1} \mathrm{H}$ NMR $\left(\mathrm{CDCl}_{3}\right): \delta 7.39-7.22(\mathrm{~m}, 5 \mathrm{H}), 4.83(\mathrm{~s}, 1 \mathrm{H}), 4.74(\mathrm{~s}, 1 \mathrm{H})$, $4.32(\mathrm{~s}, 2 \mathrm{H}), 2.08\left(\mathrm{t},{ }^{3} \mathrm{~J}_{\mathrm{HH}}=6.8 \mathrm{~Hz}, 2 \mathrm{H}\right), 1.75(\mathrm{~s}, 3 \mathrm{H}), 1.21$ (quint, $\left.{ }^{3} J_{\mathrm{HH}}=7.2 \mathrm{~Hz}, 2 \mathrm{H}\right)$, 1.05 (sextet, $\left.{ }^{3} J_{\mathrm{HH}}=7.4 \mathrm{~Hz}, 2 \mathrm{H}\right), 0.74\left(\mathrm{t},{ }^{3} \mathrm{~J}_{\mathrm{HH}}=7.3 \mathrm{~Hz}, 3 \mathrm{H}\right) .{ }^{13} \mathrm{C} \mathrm{NMR}\left(\mathrm{CDCl}_{3}\right): \delta 154.2$, $141.8,139.9,128.6,127.8,127.5,113.3,94.2,74.8,54.1,29.2,21.3,20.1,18.2,13.2$. Anal. Calcd for $\mathrm{C}_{17} \mathrm{H}_{21} \mathrm{NO}$ : C, 79.96; $\mathrm{H}, 8.29$. Found: $\mathrm{C}, 80.01 ; \mathrm{H}, 8.38$.

\section{$N-(2-M e t h y l-2-p r o p e n y l)-N-p h e n y l-5-m e t h y l-2-h e x y n a m i d e ~(11 b)$}

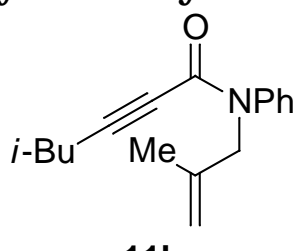

11b

This was synthesized from phenyl isocyanate and 4-methyl-1-pentyne, following the procedure for compound 11a. Pink oil, 51\% overall yield. This compound exists as a 5.9:1 mixture of rotamers.

Major rotamer: ${ }^{1} \mathrm{H}$ NMR $\left(\mathrm{CDCl}_{3}\right): \delta 7.39-7.22(\mathrm{~m}, 5 \mathrm{H}), 4.83(\mathrm{~s}, 1 \mathrm{H}), 4.75(\mathrm{~s}, 1 \mathrm{H})$, $4.33(\mathrm{~s}, 2 \mathrm{H}), 2.00\left(\mathrm{~d},{ }^{3} \mathrm{~J}_{\mathrm{HH}}=6.3 \mathrm{~Hz}, 2 \mathrm{H}\right), 1.76(\mathrm{~s}, 3 \mathrm{H}), 1.58-1.50(\mathrm{~m}, 1 \mathrm{H}), 0.68\left(\mathrm{~d},{ }^{3} \mathrm{~J}_{\mathrm{HH}}=\right.$ $6.7 \mathrm{~Hz}, 6 \mathrm{H}) .{ }^{13} \mathrm{C}$ NMR $\left(\mathrm{CDCl}_{3}\right): \delta 154.1,141.7,139.8,128.7,127.8,127.5,113.2,93.1$, 75.6, 54.0, 27.6, 27.0, 21.4, 20.0. Anal. Calcd for $\mathrm{C}_{17} \mathrm{H}_{21} \mathrm{NO}$ : C, 79.96; H, 8.29. Found: C, $79.91 ; \mathrm{H}, 8.34$.

\section{General Procedure for the Preparation of Arylzinc Chlorides}

$t$-BuLi (13.7 mL, $20.0 \mathrm{mmol} ; 1.46 \mathrm{M}$ solution in pentane) was added dropwise to a solution of aryl bromide $(10.0 \mathrm{mmol})$ in THF $(10 \mathrm{~mL})$ at $-78{ }^{\circ} \mathrm{C}$. The resulting mixture was stirred for $30 \mathrm{~min}$ at $-78{ }^{\circ} \mathrm{C}$, and then for $15 \mathrm{~min}$ at room temperature. The aryllithium solution thus obtained was added to a suspension of $\mathrm{ZnCl}_{2}(1.45 \mathrm{~g}$, $10.6 \mathrm{mmol})$ in THF $(2.5 \mathrm{~mL})$ at $0{ }^{\circ} \mathrm{C}$. The reaction mixture was stirred for $15 \mathrm{~min}$ at room temperature, and was then concentrated under vacuum to remove pentane, affording a solution of arylzinc chloride in THF. The concentration was determined by acid-base titration with methyl orange (typically $0.6-1.2 \mathrm{M}$ ).

Only 3-chlorophenylzinc chloride was prepared from 3-chlorophenylmagnesium bromide $\left(0.40 \mathrm{~mL}, 0.40 \mathrm{mmol} ; 1.00 \mathrm{M}\right.$ solution in THF) and $\mathrm{ZnCl}_{2}$ (54.5 mg, 0.40 $\mathrm{mmol})$ at $0{ }^{\circ} \mathrm{C}$ and was directly used in the rhodium-catalyzed reaction.

\section{Methylzinc Chloride}

MeLi (4.81 mL, $5.00 \mathrm{mmol} ; 1.04 \mathrm{M}$ solution in $\mathrm{Et}_{2} \mathrm{O}$ ) was added dropwise to a suspension of $\mathrm{ZnCl}_{2}(0.73 \mathrm{~g}$, $5.36 \mathrm{mmol})$ in $\mathrm{THF}(2.0 \mathrm{~mL})$ at $0{ }^{\circ} \mathrm{C}$, and the resulting 
mixture was stirred for $1 \mathrm{~h}$ at room temperature. The concentration was determined by acid-base titration with methyl orange $(0.67 \mathrm{M})$. 


\section{Catalytic Reactions}

\section{General Procedure for Table 2.}

A solution of $\left[\mathrm{RhCl}\left(\mathrm{C}_{2} \mathrm{H}_{4}\right)_{2}\right]_{2}(3.9 \mathrm{mg}, 20 \mu \mathrm{mol} \mathrm{Rh})$ and dppf $(12.2 \mathrm{mg}, 22.0 \mu \mathrm{mol})$ in dioxane $(10 \mathrm{~mL})$ was stirred for $10 \mathrm{~min}$ at room temperature. Substrate $1(0.20$ $\mathrm{mmol})$ and $\mathrm{ArZnCl}(0.33-0.60 \mathrm{~mL}, 0.40-0.60 \mathrm{mmol} ; 0.67-1.22 \mathrm{M}$ solution in THF) were added to it, and the mixture was stirred for $20 \mathrm{~h}$ at $40{ }^{\circ} \mathrm{C}$. The reaction was quenched with water $(80 \mu \mathrm{L})$, passed through a pad of silica gel with EtOAc, and concentrated under vacuum. The residue was purified by silica gel preparative TLC with $\mathrm{CH}_{2} \mathrm{Cl}_{2}$ to afford product 2 .

Entry 1. Yellow solid. 87\% yield.<smiles>CCCC(=C1C(=O)N([14CH3])c2ccccc21)c1ccccc1</smiles>

${ }^{1} \mathrm{H}$ NMR $\left(\mathrm{CDCl}_{3}\right): \delta 7.49-7.42(\mathrm{~m}, 3 \mathrm{H}), 7.26-7.23(\mathrm{~m}, 2 \mathrm{H}), 7.11\left(\mathrm{td},{ }^{3} J_{\mathrm{HH}}=7.7 \mathrm{~Hz}\right.$ and $\left.{ }^{4} J_{\mathrm{HH}}=1.0 \mathrm{~Hz}, 1 \mathrm{H}\right), 6.73\left(\mathrm{~d},{ }^{3} J_{\mathrm{HH}}=7.7 \mathrm{~Hz}, 1 \mathrm{H}\right), 6.61\left(\mathrm{t},{ }^{3} J_{\mathrm{HH}}=7.7 \mathrm{~Hz}, 1 \mathrm{H}\right), 6.01(\mathrm{~d}$, $\left.{ }^{3} \mathrm{~J}_{\mathrm{HH}}=7.8 \mathrm{~Hz}, 1 \mathrm{H}\right), 3.32\left(\mathrm{t},{ }^{3} \mathrm{~J}_{\mathrm{HH}}=7.5 \mathrm{~Hz}, 2 \mathrm{H}\right), 3.26(\mathrm{~s}, 3 \mathrm{H}), 1.51-1.38(\mathrm{~m}, 4 \mathrm{H}), 0.90(\mathrm{t}$, $\left.{ }^{3} J_{\mathrm{HH}}=7.0 \mathrm{~Hz}, 3 \mathrm{H}\right) .{ }^{13} \mathrm{C} \mathrm{NMR}\left(\mathrm{CDCl}_{3}\right): \delta 167.8,159.7,142.3,141.7,129.0,128.2,128.0$, $126.9,123.2,122.8,122.7,121.3,107.3,34.8,29.8,25.7,22.9,13.9$. Anal. Calcd for $\mathrm{C}_{20} \mathrm{H}_{21} \mathrm{NO}$ : C, 82.44; H, 7.26. Found: $\mathrm{C}, 82.27 ; \mathrm{H}, 7.28$.

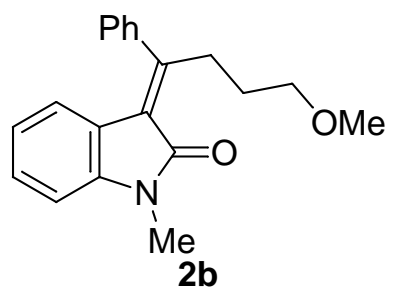

Entry 3. Yellow solid. $84 \%$ yield.

${ }^{1} \mathrm{H} \mathrm{NMR}\left(\mathrm{CDCl}_{3}\right): \delta$ 7.49-7.41 (m, 3H), 7.27-7.22 (m, 2H), $7.12\left(\mathrm{t},{ }^{3} \mathrm{~J}_{\mathrm{HH}}=7.6 \mathrm{~Hz}, 1 \mathrm{H}\right)$, $6.74\left(\mathrm{~d},{ }^{3} J_{\mathrm{HH}}=7.8 \mathrm{~Hz}, 1 \mathrm{H}\right), 6.62\left(\mathrm{t},{ }^{3} \mathrm{~J}_{\mathrm{HH}}=7.7 \mathrm{~Hz}, 1 \mathrm{H}\right), 6.04\left(\mathrm{~d},{ }^{3} J_{\mathrm{HH}}=7.7 \mathrm{~Hz}, 1 \mathrm{H}\right), 3.48$ $\left(\mathrm{t},{ }^{3} \mathrm{~J}_{\mathrm{HH}}=6.8 \mathrm{~Hz}, 2 \mathrm{H}\right), 3.40-3.33(\mathrm{~m}, 2 \mathrm{H}), 3.29(\mathrm{~s}, 3 \mathrm{H}), 3.26(\mathrm{~s}, 3 \mathrm{H}), 1.82-1.74(\mathrm{~m}, 2 \mathrm{H})$. ${ }^{13} \mathrm{C}$ NMR $\left(\mathrm{CDCl}_{3}\right): \delta 167.7,158.7,142.3,141.3,129.1,128.3,128.1,126.9,123.5,122.9$, 122.6, 121.3, 107.4, 72.5, 58.5, 31.6, 27.6, 25.7. Anal. Calcd for $\mathrm{C}_{20} \mathrm{H}_{21} \mathrm{NO}_{2}: \mathrm{C}, 78.15 ; \mathrm{H}$, 6.89. Found: C, 78.09; H, 7.03.

Entry 4. Yellow solid. 94\% yield.<smiles>CC(C)C/C(=C1/C(=O)N(C(C)(C)C)c2ccccc21)c1ccccc1</smiles>

${ }^{1} \mathrm{H}$ NMR $\left(\mathrm{CDCl}_{3}\right): \delta 7.50-7.42(\mathrm{~m}, 3 \mathrm{H}), 7.28-7.23(\mathrm{~m}, 2 \mathrm{H}), 7.12\left(\mathrm{td},{ }^{3} J_{\mathrm{HH}}=7.7 \mathrm{~Hz}\right.$ and $\left.{ }^{4} J_{\mathrm{HH}}=1.1 \mathrm{~Hz}, 1 \mathrm{H}\right), 6.73\left(\mathrm{~d},{ }^{3} J_{\mathrm{HH}}=7.8 \mathrm{~Hz}, 1 \mathrm{H}\right), 6.60\left(\mathrm{td},{ }^{3} J_{\mathrm{HH}}=7.7 \mathrm{~Hz}\right.$ and ${ }^{4} J_{\mathrm{HH}}=$ 
$1.1 \mathrm{~Hz}, 1 \mathrm{H}), 6.01\left(\mathrm{~d},{ }^{3} \mathrm{~J}_{\mathrm{HH}}=7.7 \mathrm{~Hz}, 1 \mathrm{H}\right), 3.34\left(\mathrm{~d},{ }^{3} \mathrm{~J}_{\mathrm{HH}}=7.3 \mathrm{~Hz}, 2 \mathrm{H}\right), 3.26(\mathrm{~s}, 3 \mathrm{H}), 1.69-$ $1.78(\mathrm{~m}, 1 \mathrm{H}), 0.98\left(\mathrm{~d},{ }^{3} J_{\mathrm{HH}}=6.7 \mathrm{~Hz}, 6 \mathrm{H}\right) .{ }^{13} \mathrm{C} \mathrm{NMR}\left(\mathrm{CDCl}_{3}\right): \delta 168.0,158.8,142.2$, 141.5, 129.0, 128.2, 128.1, 127.0, 124.3, 122.9, 122.8, 121.3, 107.3, 42.6, 27.1, 25.7, 22.3. Anal. Calcd for $\mathrm{C}_{20} \mathrm{H}_{21} \mathrm{NO}$ : C, 82.44; H, 7.26. Found: C, 82.28; H, 7.34.

Entry 5. Yellow solid. $57 \%$ yield.

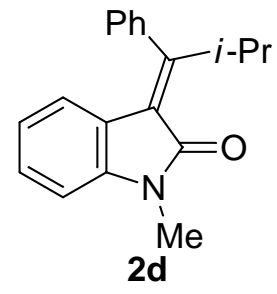

${ }^{1} \mathrm{H}$ NMR $\left(\mathrm{CDCl}_{3}\right): \delta$ 7.51-7.43 (m, 3H), 7.15-7.10 (m, 2H), $7.10\left(\mathrm{td},{ }^{3} J_{\mathrm{HH}}=7.6 \mathrm{~Hz}\right.$ and $\left.{ }^{4} J_{\mathrm{HH}}=1.0 \mathrm{~Hz}, 1 \mathrm{H}\right), 6.72\left(\mathrm{~d},{ }^{3} J_{\mathrm{HH}}=7.8 \mathrm{~Hz}, 1 \mathrm{H}\right), 6.60\left(\mathrm{td},{ }^{3} J_{\mathrm{HH}}=7.7 \mathrm{~Hz}\right.$ and ${ }^{4} J_{\mathrm{HH}}=$ $0.8 \mathrm{~Hz}, 1 \mathrm{H}), 5.58\left(\mathrm{~d},{ }^{3} J_{\mathrm{HH}}=7.8 \mathrm{~Hz}, 1 \mathrm{H}\right), 5.01$ (septet, $\left.{ }^{3} J_{\mathrm{HH}}=6.9 \mathrm{~Hz}, 1 \mathrm{H}\right), 3.26(\mathrm{~s}, 3 \mathrm{H})$, $1.07\left(\mathrm{~d},{ }^{3} J_{\mathrm{HH}}=6.8 \mathrm{~Hz}, 6 \mathrm{H}\right) .{ }^{13} \mathrm{C} \mathrm{NMR}\left(\mathrm{CDCl}_{3}\right): \delta 167.7,164.3,142.3,138.0,128.7,127.9$, 127.7, 127.3, 123.2, 123.0, 122.7, 121.4, 107.2, 28.4, 25.7, 20.7. Anal. Calcd for $\mathrm{C}_{19} \mathrm{H}_{19} \mathrm{NO}: \mathrm{C}, 82.28 ; \mathrm{H}, 6.90$. Found: $\mathrm{C}, 82.03 ; \mathrm{H}, 6.90$.

Entry 6. Yellow solid. $85 \%$ yield.

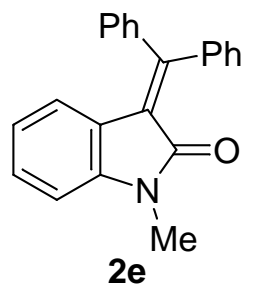

${ }^{1} \mathrm{H}$ NMR $\left(\mathrm{CDCl}_{3}\right): \delta$ 7.46-7.39 $(\mathrm{m}, 3 \mathrm{H}), 7.38-7.30(\mathrm{~m}, 7 \mathrm{H}), 7.16\left(\mathrm{t},{ }^{3} J_{\mathrm{HH}}=7.7 \mathrm{~Hz}, 1 \mathrm{H}\right)$, $6.76\left(\mathrm{~d},{ }^{3} J_{\mathrm{HH}}=7.8 \mathrm{~Hz}, 1 \mathrm{H}\right), 6.68\left(\mathrm{t},{ }^{3} J_{\mathrm{HH}}=7.7 \mathrm{~Hz}, 1 \mathrm{H}\right), 6.42\left(\mathrm{~d},{ }^{3} J_{\mathrm{HH}}=7.7 \mathrm{~Hz}, 1 \mathrm{H}\right), 3.20$ $(\mathrm{s}, 3 \mathrm{H}) .{ }^{13} \mathrm{C} \mathrm{NMR}\left(\mathrm{CDCl}_{3}\right): \delta 166.8,154.5,143.3,141.3,139.9,129.9,129.3,129.1,129.0$, $128.9,128.7,127.8,124.2,123.2,123.1,121.3,107.6,25.8$. Anal. Calcd for $\mathrm{C}_{22} \mathrm{H}_{17} \mathrm{NO}$ : C, 84.86; H, 5.50. Found: C, 85.16; H, 5.78.

Entry 7. Yellow solid. 77\% yield.

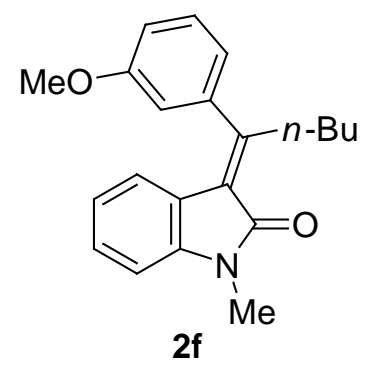

${ }^{1} \mathrm{H} \mathrm{NMR}\left(\mathrm{CDCl}_{3}\right): \delta 7.39\left(\mathrm{t},{ }^{3} J_{\mathrm{HH}}=7.9 \mathrm{~Hz}, 1 \mathrm{H}\right), 7.12\left(\mathrm{td},{ }^{3} J_{\mathrm{HH}}=7.7 \mathrm{~Hz}\right.$ and ${ }^{4} J_{\mathrm{HH}}=$ $0.9 \mathrm{~Hz}, 1 \mathrm{H}), 6.98\left(\mathrm{dd},{ }^{3} J_{\mathrm{HH}}=8.0 \mathrm{~Hz}\right.$ and $\left.{ }^{4} J_{\mathrm{HH}}=2.3 \mathrm{~Hz}, 1 \mathrm{H}\right), 6.82\left(\mathrm{~d},{ }^{3} J_{\mathrm{HH}}=7.4 \mathrm{~Hz}, 1 \mathrm{H}\right)$, $6.77\left(\mathrm{dd},{ }^{4} J_{\mathrm{HH}}=2.4\right.$ and $\left.1.5 \mathrm{~Hz}, 1 \mathrm{H}\right), 6.74\left(\mathrm{~d},{ }^{3} J_{\mathrm{HH}}=7.7 \mathrm{~Hz} 1 \mathrm{H}\right), 6.64\left(\mathrm{td},{ }^{3} J_{\mathrm{HH}}=7.6 \mathrm{~Hz}\right.$ and $\left.{ }^{4} J_{\mathrm{HH}}=0.8 \mathrm{~Hz}, 1 \mathrm{H}\right), 6.11\left(\mathrm{~d},{ }^{3} J_{\mathrm{HH}}=7.5 \mathrm{~Hz}, 1 \mathrm{H}\right), 3.82(\mathrm{~s}, 3 \mathrm{H}), 3.41-3.33(\mathrm{~m}, 1 \mathrm{H}), 3.26$ $(\mathrm{s}, 3 \mathrm{H}), 3.27-3.18(\mathrm{~m}, 1 \mathrm{H}), 1.52-1.38(\mathrm{~m}, 4 \mathrm{H}), 0.91\left(\mathrm{t},{ }^{3} J_{\mathrm{HH}}=7.1 \mathrm{~Hz}, 3 \mathrm{H}\right) .{ }^{13} \mathrm{C} \mathrm{NMR}$ $\left(\mathrm{CDCl}_{3}\right): \delta 167.8,160.1,159.4,143.0,142.3,130.2,128.0,123.1,123.0,122.6,121.4,119.1$, 113.7, 112.1, 107.3, 55.3, 34.7, 29.8, 25.7, 22.9, 13.9. Anal. Calcd for $\mathrm{C}_{21} \mathrm{H}_{23} \mathrm{NO}_{2}$ : C, 
78.47; H, 7.21. Found: C, 78.49; H, 7.36.

Entry 8. Yellow solid. 80\% yield.

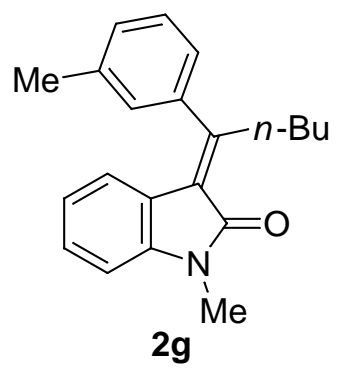

${ }^{1} \mathrm{H} \mathrm{NMR}\left(\mathrm{CDCl}_{3}\right): \delta 7.35\left(\mathrm{t},{ }^{3} J_{\mathrm{HH}}=7.5 \mathrm{~Hz}, 1 \mathrm{H}\right), 7.25\left(\mathrm{~d},{ }^{3} J_{\mathrm{HH}}=7.5 \mathrm{~Hz}, 1 \mathrm{H}\right), 6.98(\mathrm{td}$, ${ }^{3} J_{\mathrm{HH}}=7.7 \mathrm{~Hz}$ and $\left.{ }^{4} J_{\mathrm{HH}}=1.1 \mathrm{~Hz}, 1 \mathrm{H}\right), 7.05(\mathrm{~s}, 1 \mathrm{H}), 7.03\left(\mathrm{~d},{ }^{3} J_{\mathrm{HH}}=7.9 \mathrm{~Hz}, 1 \mathrm{H}\right), 6.73(\mathrm{~d}$, $\left.{ }^{3} J_{\mathrm{HH}}=7.7 \mathrm{~Hz}, 1 \mathrm{H}\right), 6.62\left(\mathrm{td},{ }^{3} \mathrm{~J}_{\mathrm{HH}}=7.7 \mathrm{~Hz}\right.$ and $\left.{ }^{4} \mathrm{~J}_{\mathrm{HH}}=1.0 \mathrm{~Hz}, 1 \mathrm{H}\right), 6.05\left(\mathrm{~d},{ }^{3} \mathrm{~J}_{\mathrm{HH}}=7.7\right.$ $\mathrm{Hz}, 1 \mathrm{H}), 3.41-3.33(\mathrm{~m}, 1 \mathrm{H}), 3.26(\mathrm{~s}, 3 \mathrm{H}), 3.27-3.18(\mathrm{~m}, 1 \mathrm{H}), 2.40(\mathrm{~s}, 3 \mathrm{H}), 1.52-1.38(\mathrm{~m}$, $4 \mathrm{H}), 0.91\left(\mathrm{t},{ }^{3} \mathrm{~J}_{\mathrm{HH}}=7.1 \mathrm{~Hz}, 3 \mathrm{H}\right) .{ }^{13} \mathrm{C} \mathrm{NMR}\left(\mathrm{CDCl}_{3}\right): \delta 167.9,160.1,142.2,141.6,138.7$, $128.9,128.8,127.9,127.2,123.9,123.0,122.9,122.8,121.3,107.3,34.8,29.8,25.6,23.0$, 21.5, 13.9. Anal. Calcd for $\mathrm{C}_{21} \mathrm{H}_{23} \mathrm{NO}$ : $\mathrm{C}, 82.58 ; \mathrm{H}, 7.59$. Found: $\mathrm{C}, 82.30 ; \mathrm{H}, 7.58$.<smiles>CCCCOC(=O)C1C(=O)N(C)c2ccccc21</smiles>

Entry 9. Yellow solid. 78\% yield (contaminated with $\sim 5 \%$ impurity).

${ }^{1} \mathrm{H}$ NMR $\left(\mathrm{CDCl}_{3}\right): \delta$ 7.45-7.29 (m, 2H), 7.27-7.24 (m, 1H), 7.16-7.11 (m, 2H), $6.74(\mathrm{~d}$, $\left.{ }^{3} J_{\mathrm{HH}}=7.7 \mathrm{~Hz}, 1 \mathrm{H}\right), 6.66\left(\mathrm{td},{ }^{3} J_{\mathrm{HH}}=7.7 \mathrm{~Hz}\right.$ and $\left.{ }^{4} J_{\mathrm{HH}}=1.0 \mathrm{~Hz}, 1 \mathrm{H}\right), 6.06\left(\mathrm{~d},{ }^{3} J_{\mathrm{HH}}=7.7\right.$ $\mathrm{Hz}, 1 \mathrm{H}), 3.40-3.32(\mathrm{~m}, 1 \mathrm{H}), 3.26(\mathrm{~s}, 3 \mathrm{H}), 3.27-3.16(\mathrm{~m}, 1 \mathrm{H}), 1.50-1.38(\mathrm{~m}, 4 \mathrm{H}), 0.90(\mathrm{t}$, $\left.{ }^{3} \mathrm{~J}_{\mathrm{HH}}=7.6 \mathrm{~Hz}, 3 \mathrm{H}\right) .{ }^{13} \mathrm{C} \mathrm{NMR}\left(\mathrm{CDCl}_{3}\right): \delta 167.6,157.3,143.3,142.4,134.9,130.4,128.4$, $128.3,127.0,125.2,123.6,122.8,122.3,121.4,107.5,34.5,29.7,25.7,22.9,13.9$. Anal. Calcd for $\mathrm{C}_{20} \mathrm{H}_{20} \mathrm{ClNO}$ : C, 73.72; $\mathrm{H}, 6.19$. Found: C, 73.99; H, 6.13.

Entry 10. Yellow solid. $77 \%$ yield.

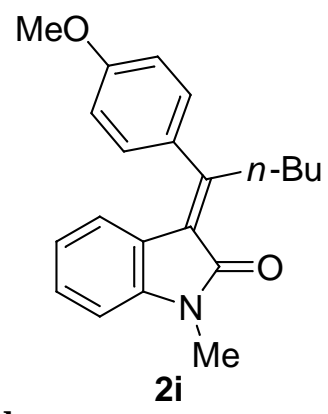

${ }^{1} \mathrm{H} \mathrm{NMR}\left(\mathrm{CDCl}_{3}\right): \delta 7.19\left(\mathrm{~d},{ }^{3} J_{\mathrm{HH}}=8.5 \mathrm{~Hz}, 2 \mathrm{H}\right), 7.12\left(\mathrm{t},{ }^{3} \mathrm{~J}_{\mathrm{HH}}=7.6 \mathrm{~Hz}, 1 \mathrm{H}\right), 6.99(\mathrm{~d}$, $\left.{ }^{3} \mathrm{~J}_{\mathrm{HH}}=8.5 \mathrm{~Hz}, 2 \mathrm{H}\right), 6.73\left(\mathrm{~d},{ }^{3} \mathrm{~J}_{\mathrm{HH}}=7.7 \mathrm{~Hz}, 1 \mathrm{H}\right), 6.65\left(\mathrm{t},{ }^{3} \mathrm{~J}_{\mathrm{HH}}=7.6 \mathrm{~Hz}, 1 \mathrm{H}\right), 6.22\left(\mathrm{~d},{ }^{3} \mathrm{~J}_{\mathrm{HH}}\right.$ $=7.7 \mathrm{~Hz} 1 \mathrm{H}), 3.89(\mathrm{~s}, 3 \mathrm{H}), 3.32-3.25(\mathrm{~m}, 2 \mathrm{H}), 3.26(\mathrm{~s}, 3 \mathrm{H}), 1.50-1.37(\mathrm{~m}, 4 \mathrm{H}), 0.89(\mathrm{t}$, $\left.{ }^{3} J_{\mathrm{HH}}=6.9 \mathrm{~Hz}, 3 \mathrm{H}\right) .{ }^{13} \mathrm{C} \mathrm{NMR}\left(\mathrm{CDCl}_{3}\right): \delta 167.9,160.0,159.6,142.2,133.8,128.5,127.8$, $123.1,122.9,122.7,121.2,114.3,107.3,55.3,35.0,30.0,25.6,22.9,13.9$. Anal. Calcd for 
$\mathrm{C}_{21} \mathrm{H}_{23} \mathrm{NO}_{2}$ : C, 78.47; H, 7.21. Found: C, 78.51; H, 7.27.

Entry 11. Yellow solid. $75 \%$ yield.

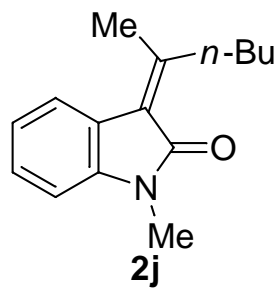

${ }^{1} \mathrm{H}$ NMR $\left(\mathrm{CDCl}_{3}\right): \delta 7.52\left(\mathrm{~d},{ }^{3} J_{\mathrm{HH}}=7.5 \mathrm{~Hz}, 1 \mathrm{H}\right), 7.24\left(\mathrm{td},{ }^{3} \mathrm{~J}_{\mathrm{HH}}=8.0 \mathrm{~Hz}\right.$ and ${ }^{4} J_{\mathrm{HH}}=$ $1.1 \mathrm{~Hz}, 1 \mathrm{H}), 7.03\left(\mathrm{td},{ }^{3} J_{\mathrm{HH}}=7.6 \mathrm{~Hz}\right.$ and $\left.{ }^{4} J_{\mathrm{HH}}=1.0 \mathrm{~Hz}, 1 \mathrm{H}\right), 6.80\left(\mathrm{~d},{ }^{3} J_{\mathrm{HH}}=7.7 \mathrm{~Hz}, 1 \mathrm{H}\right)$, $3.24(\mathrm{~s}, 3 \mathrm{H}), 3.13-3.09(\mathrm{~m}, 2 \mathrm{H}), 2.36(\mathrm{~s}, 3 \mathrm{H}), 1.58-1.50(\mathrm{~m}, 2 \mathrm{H}), 1.50-1.40(\mathrm{~m}, 2 \mathrm{H}), 0.96$ $\left(\mathrm{t},{ }^{3} \mathrm{~J}_{\mathrm{HH}}=7.3 \mathrm{~Hz}, 3 \mathrm{H}\right) .{ }^{13} \mathrm{C} \mathrm{NMR}\left(\mathrm{CDCl}_{3}\right): \delta 167.5,159.5,142.1,127.4,123.7,123.4$, $122.4,121.5,107.4,35.5,30.4,25.5,23.5,23.0,14.0$. Anal. Calcd for $\mathrm{C}_{15} \mathrm{H}_{19} \mathrm{NO}_{2}$ : C, 78.56; H, 8.35. Found: C, 78.45; H, 8.55.

\section{Procedure for Equation 2.}

A solution of $\left[\mathrm{RhCl}\left(\mathrm{C}_{2} \mathrm{H}_{4}\right)_{2}\right]_{2}(3.9 \mathrm{mg}, 20 \mu \mathrm{mol} \mathrm{Rh})$ and dppf $(12.2 \mathrm{mg}, 22.0 \mu \mathrm{mol})$ in dioxane $(10 \mathrm{~mL})$ was stirred for $10 \mathrm{~min}$ at room temperature. 1a $(68.2 \mathrm{mg}, 0.20$ $\mathrm{mmol}), 3$ (55.0 mg, $0.20 \mathrm{mmol})$, and $\mathrm{PhZnCl}(0.41 \mathrm{~mL}, 0.40 \mathrm{mmol} ; 0.97 \mathrm{M}$ solution in THF) were added to it, and the mixture was stirred for $20 \mathrm{~h}$ at $40{ }^{\circ} \mathrm{C}$. The reaction was quenched with water $(80 \mu \mathrm{L})$, passed through a pad of silica gel with EtOAc, and concentrated under vacuum. The residue was purified by silica gel preparative TLC with $\mathrm{CH}_{2} \mathrm{Cl}_{2}$ to afford product $2 \mathrm{a}$ as a yellow solid $(47.3 \mathrm{mg}, 0.162 \mathrm{mmol} ; 81 \%$ yield) and 3 was recovered unchanged (82\%).

\section{Procedures for Equation 3.}

Reaction with a palladium catalyst: $\mathrm{PhZnCl}(0.41 \mathrm{~mL}, 0.40 \mathrm{mmol}$; $0.97 \mathrm{M}$ solution in THF) was added to a solution of $\mathrm{Pd}\left(\mathrm{PPh}_{3}\right)_{4}(11.6 \mathrm{mg}, 10.0 \mu \mathrm{mol})$ and $5(68.2 \mathrm{mg}$, $0.20 \mathrm{mmol})$ in THF $(1.0 \mathrm{~mL})$, and the mixture was stirred for $3 \mathrm{~h}$ at $60{ }^{\circ} \mathrm{C}$. The reaction was quenched with water $(80 \mu \mathrm{L})$, passed through a pad of silica gel with EtOAc, and concentrated under vacuum. The residue was purified by silica gel preparative TLC with $\mathrm{Et}_{2} \mathrm{O} /$ hexane $=1 / 1$ to afford product 6 (56.5 mg, $0.194 \mathrm{mmol}$; 97\% yield).

Reaction with a rhodium catalyst: A solution of $\left[\mathrm{RhCl}\left(\mathrm{C}_{2} \mathrm{H}_{4}\right)_{2}\right]_{2}(3.9 \mathrm{mg}, 20 \mu \mathrm{mol}$ $\mathrm{Rh})$ and dppf $(12.2 \mathrm{mg}, 22.0 \mu \mathrm{mol})$ in dioxane $(10 \mathrm{~mL})$ was stirred for $10 \mathrm{~min}$ at room temperature. $5(68.2 \mathrm{mg}, 0.20 \mathrm{mmol})$ and $\mathrm{PhZnCl}(0.41 \mathrm{~mL}, 0.40 \mathrm{mmol} ; 0.97 \mathrm{M}$ solution in THF) were added to it, and the mixture was stirred for $20 \mathrm{~h}$ at $40{ }^{\circ} \mathrm{C}$. The reaction was quenched with water $(80 \mu \mathrm{L})$, passed through a pad of silica gel with EtOAc, and concentrated under vacuum. The residue was purified by silica gel preparative TLC with $\mathrm{CH}_{2} \mathrm{Cl}_{2}$ to afford product 7 as a pale yellow oil (44 $\mu \mathrm{mol} ; 22 \%$ yield, a mixture of $E / Z$ isomers).

\section{Procedure for Equation 4.}

A solution of $\left[\mathrm{RhCl}\left(\mathrm{C}_{2} \mathrm{H}_{4}\right)_{2}\right]_{2}(1.9 \mathrm{mg}, 10 \mu \mathrm{mol} \mathrm{Rh})$ and dppf $(6.1 \mathrm{mg}, 11 \mu \mathrm{mol})$ in dioxane $(5.0 \mathrm{~mL})$ was stirred for $10 \mathrm{~min}$ at room temperature. Substrate $8(46.7 \mathrm{mg}$, $0.10 \mathrm{mmol})$ and $\mathrm{PhZnCl}(0.20 \mathrm{~mL}, 0.19 \mathrm{mmol} ; 0.97 \mathrm{M}$ solution in THF) were added to it, and the mixture was stirred for $20 \mathrm{~h}$ at $40{ }^{\circ} \mathrm{C}$. The reaction was quenched with water $(40 \mu \mathrm{L})$, passed through a pad of silica gel with EtOAc, and concentrated under vacuum. The residue was purified by silica gel preparative TLC with $\mathrm{CH}_{2} \mathrm{Cl}_{2}$ 
to afford a mixture of $\mathbf{9}$ and $\mathbf{1 0}$ as a yellow solid (30.7 mg, $74.0 \mu \mathrm{mol} ; 74 \%$ yield $(\mathbf{9} / \mathbf{1 0}$ $=95 / 5)$ ). Analytically pure $\mathbf{9}$ and $\mathbf{1 0}$ were obtained by further chromatographic purification on silica gel.<smiles>CCCCC(=C1C(=O)N(C)c2ccc(I)cc21)c1ccccc1</smiles><smiles>CCCC(=C1C(=O)N(C)c2ccc(-c3ccccc3)cc21)c1ccccc1</smiles>

9: ${ }^{1} \mathrm{H}$ NMR $\left(\mathrm{CDCl}_{3}\right): \delta 7.54-7.47(\mathrm{~m}, 3 \mathrm{H}), 7.41\left(\mathrm{dd},{ }^{3} J_{\mathrm{HH}}=8.1 \mathrm{~Hz}\right.$ and ${ }^{4} J_{\mathrm{HH}}=1.6 \mathrm{~Hz}$, $1 \mathrm{H}), 7.22-7.19(\mathrm{~m}, 2 \mathrm{H}), 6.51\left(\mathrm{~d},{ }^{3} J_{\mathrm{HH}}=8.1 \mathrm{~Hz}, 1 \mathrm{H}\right), 6.19\left(\mathrm{~d},{ }^{4} J_{\mathrm{HH}}=1.7 \mathrm{~Hz}, 1 \mathrm{H}\right), 3.35-$ $3.28(\mathrm{~m}, 2 \mathrm{H}), 3.23(\mathrm{~s}, 3 \mathrm{H}), 1.50-1.38(\mathrm{~m}, 4 \mathrm{H}), 0.90\left(\mathrm{t},{ }^{3} \mathrm{~J}_{\mathrm{HH}}=7.1 \mathrm{~Hz}, 3 \mathrm{H}\right) .{ }^{13} \mathrm{C} \mathrm{NMR}$ $\left(\mathrm{CDCl}_{3}\right): \delta 167.1,161.7,141.7,141.0,136.3,131.7,129.1,128.6,126.6,124.9,122.3,109.3$, 84.0, 34.8, 29.7, 25.7, 22.9, 13.9. Anal. Calcd for $\mathrm{C}_{20} \mathrm{H}_{20} \mathrm{INO}$ : C, 57.57; $\mathrm{H}, 4.83$. Found: C, $57.71 ; \mathrm{H}, 4.90$.

10: ${ }^{1} \mathrm{H}$ NMR $\left(\mathrm{CDCl}_{3}\right): \delta 7.53-7.43(\mathrm{~m}, 3 \mathrm{H}), 7.37\left(\mathrm{dd},{ }^{3} J_{\mathrm{HH}}=8.1 \mathrm{~Hz}\right.$ and ${ }^{4} J_{\mathrm{HH}}=1.8$ $\mathrm{Hz}, 1 \mathrm{H}), 7.31-7.26(\mathrm{~m}, 4 \mathrm{H}), 7.23-7.19(\mathrm{~m}, 1 \mathrm{H}), 7.17-7.14(\mathrm{~m}, 2 \mathrm{H}), 6.81\left(\mathrm{~d},{ }^{3} \mathrm{~J}_{\mathrm{HH}}=7.0 \mathrm{~Hz}\right.$, $1 \mathrm{H}), 6.24\left(\mathrm{~d},{ }^{4} J_{\mathrm{HH}}=1.7 \mathrm{~Hz}, 1 \mathrm{H}\right), 3.37-3.32(\mathrm{~m}, 2 \mathrm{H}), 3.29(\mathrm{~s}, 3 \mathrm{H}), 1.55-1.40(\mathrm{~m}, 4 \mathrm{H}), 0.92$ $\left(\mathrm{t},{ }^{3} \mathrm{~J}_{\mathrm{HH}}=7.2 \mathrm{~Hz}, 3 \mathrm{H}\right) .{ }^{13} \mathrm{C} \mathrm{NMR}\left(\mathrm{CDCl}_{3}\right): \delta 167.9,160.2,141.7,140.8,134.1,129.1$, $128.5,128.2,126.9,126.6,126.5,126.2,123.3,123.2,121.8,107.5,34.6,29.8,25.8,22.9$, 13.9. Anal. Calcd for $\mathrm{C}_{26} \mathrm{H}_{25} \mathrm{NO}$ : $\mathrm{C}, 84.98 ; \mathrm{H}, 6.86$. Found: $\mathrm{C}, 84.90 ; \mathrm{H}, 7.10$.

\section{General Procedure for Equation 5.}

A solution of $\left[\mathrm{RhCl}\left(\mathrm{C}_{2} \mathrm{H}_{4}\right)_{2}\right]_{2}(3.9 \mathrm{mg}, 20 \mu \mathrm{mol} \mathrm{Rh})$ and dppf $(12.2 \mathrm{mg}, 22.0 \mu \mathrm{mol})$ in toluene $(10 \mathrm{~mL})$ was stirred for $10 \mathrm{~min}$ at room temperature. Substrate $11(0.20$ $\mathrm{mmol})$, iodomethane (141 mg, $1.00 \mathrm{mmol})$, and $\mathrm{PhZnCl}(0.66 \mathrm{~mL}, 0.60 \mathrm{mmol} ; 0.91 \mathrm{M}$ solution in THF) were added to it, and the mixture was stirred for $20 \mathrm{~h}$ at $40{ }^{\circ} \mathrm{C}$. The reaction was quenched with water $(80 \mu \mathrm{L})$, passed through a pad of silica gel with EtOAc, and concentrated under vacuum. The residue was purified by silica gel preparative TLC with $\mathrm{Et}_{2} \mathrm{O} /$ hexane $=1 / 3$ to afford product 12 (contaminated with 13).
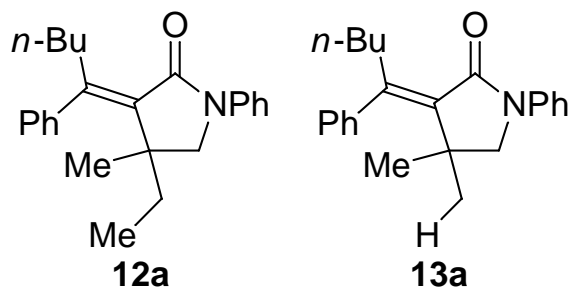

$\mathbf{R}=n \mathbf{B u}$. White solid. $70 \%$ yield $(\mathbf{1 2} \mathbf{a} / \mathbf{1 3} \mathbf{a}=96 / 4)$.

12a: ${ }^{1} \mathrm{H}$ NMR $\left(\mathrm{CDCl}_{3}\right): \delta$ 7.72-7.67 (m, 2H), 7.41-7.28 (m, 5H), 7.17-7.12 (m, 3H), $3.50\left(\mathrm{~d},{ }^{2} J_{\mathrm{HH}}=9.4 \mathrm{~Hz}, 1 \mathrm{H}\right), 3.38\left(\mathrm{~d},{ }^{2} J_{\mathrm{HH}}=9.4 \mathrm{~Hz}, 1 \mathrm{H}\right), 3.23-3.20(\mathrm{~m}, 1 \mathrm{H}), 2.90-2.82(\mathrm{~m}$, $1 \mathrm{H}), 1.47-1.32(\mathrm{~m}, 5 \mathrm{H}), 1.28-1.18(\mathrm{~m}, 1 \mathrm{H}), 0.86\left(\mathrm{t},{ }^{3} J_{\mathrm{HH}}=7.2 \mathrm{~Hz}, 3 \mathrm{H}\right), 0.85(\mathrm{~s}, 3 \mathrm{H}), 0.82$ $\left(\mathrm{t},{ }^{3} \mathrm{~J}_{\mathrm{HH}}=7.4 \mathrm{~Hz}, 3 \mathrm{H}\right) .{ }^{13} \mathrm{C} \mathrm{NMR}\left(\mathrm{CDCl}_{3}\right): \delta 168.4,153.0,142.0,140.0,134.0,128.7$, $127.8,127.6,127.0,124.3,120.0,57.4,41.0,35.4,34.1,30.3,26.7,22.9,14.0,9.0$. HRMS (ESI) calcd for $\mathrm{C}_{24} \mathrm{H}_{30} \mathrm{NO}\left(\mathrm{M}+\mathrm{H}^{+}\right)$348.2322, found 348.2321. 

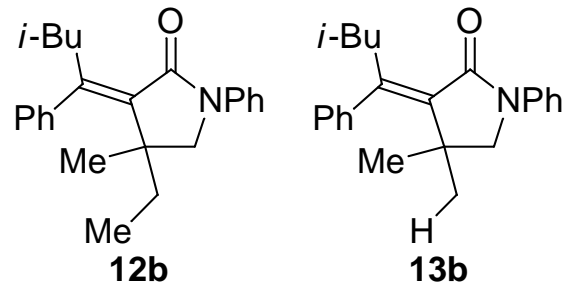

$\mathbf{R}=\boldsymbol{i B u}$. White solid. $84 \%$ yield $(\mathbf{1 2 b} / \mathbf{1 3 b}=97 / 3)$.

12b: ${ }^{1} \mathrm{H}$ NMR $\left(\mathrm{CDCl}_{3}\right): \delta$ 7.70-7.66 $(\mathrm{m}, 2 \mathrm{H}), 7.40-7.27(\mathrm{~m}, 5 \mathrm{H}), 7.17-7.12(\mathrm{~m}, 3 \mathrm{H})$, $3.48\left(\mathrm{~d},{ }^{2} J_{\mathrm{HH}}=9.4 \mathrm{~Hz}, 1 \mathrm{H}\right), 3.37\left(\mathrm{~d},{ }^{2} J_{\mathrm{HH}}=9.4 \mathrm{~Hz}, 1 \mathrm{H}\right), 3.34\left(\mathrm{dd},{ }^{2} J_{\mathrm{HH}}=12.5 \mathrm{~Hz}\right.$ and $\left.{ }^{3} J_{\mathrm{HH}}=8.2 \mathrm{~Hz}, 1 \mathrm{H}\right), 2.85\left(\mathrm{dd},{ }^{2} J_{\mathrm{HH}}=12.5 \mathrm{~Hz}\right.$ and $\left.{ }^{3} J_{\mathrm{HH}}=6.6 \mathrm{~Hz}, 1 \mathrm{H}\right), 1.63-1.52(\mathrm{~m}, 1 \mathrm{H})$, $1.49-1.40(\mathrm{~m}, 1 \mathrm{H}), 1.29-1.18(\mathrm{~m}, 1 \mathrm{H}), 0.98\left(\mathrm{~d},{ }^{3} J_{\mathrm{HH}}=6.7 \mathrm{~Hz}, 3 \mathrm{H}\right), 0.94\left(\mathrm{~d},{ }^{3} J_{\mathrm{HH}}=6.6 \mathrm{~Hz}\right.$, $3 \mathrm{H}), 0.83(\mathrm{~s}, 3 \mathrm{H}), 0.83\left(\mathrm{t},{ }^{3} \mathrm{~J}_{\mathrm{HH}}=7.5 \mathrm{~Hz}, 3 \mathrm{H}\right) .{ }^{13} \mathrm{C} \mathrm{NMR}\left(\mathrm{CDCl}_{3}\right): \delta 168.4,151.9,141.7$, 139.7, 135.3, 128.7, 128.1, 127.5, 127.0, 124.3, 120.1, 57.4, 42.6, 41.1, 34.4, 26.8, 26.4, 22.5, 21.9, 9.1. HRMS (ESI) calcd for $\mathrm{C}_{24} \mathrm{H}_{30} \mathrm{NO}\left(\mathrm{M}+\mathrm{H}^{+}\right) 348.2322$, found 348.2326. 
IV. ${ }^{1} \mathrm{H}$ NMR Spectra
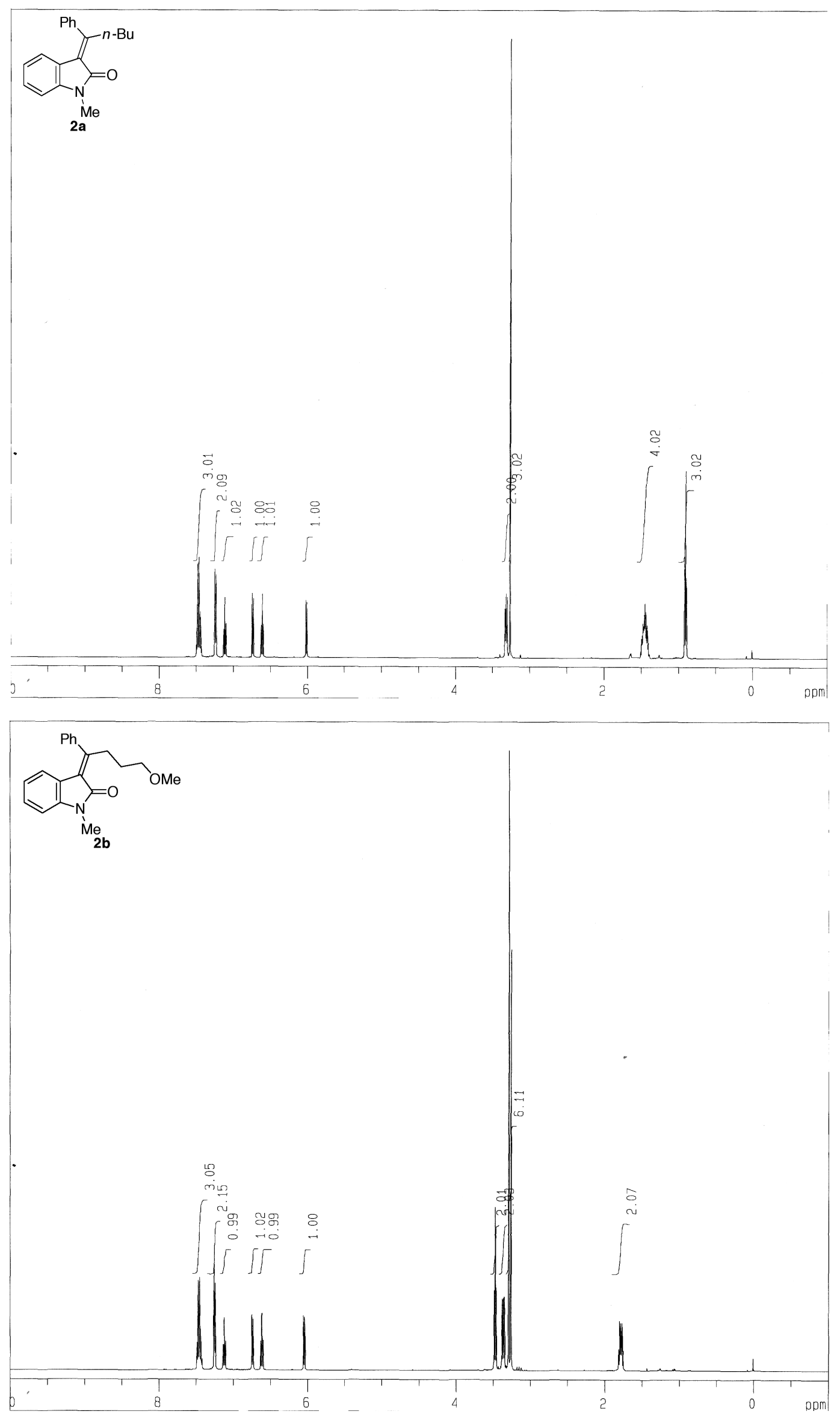

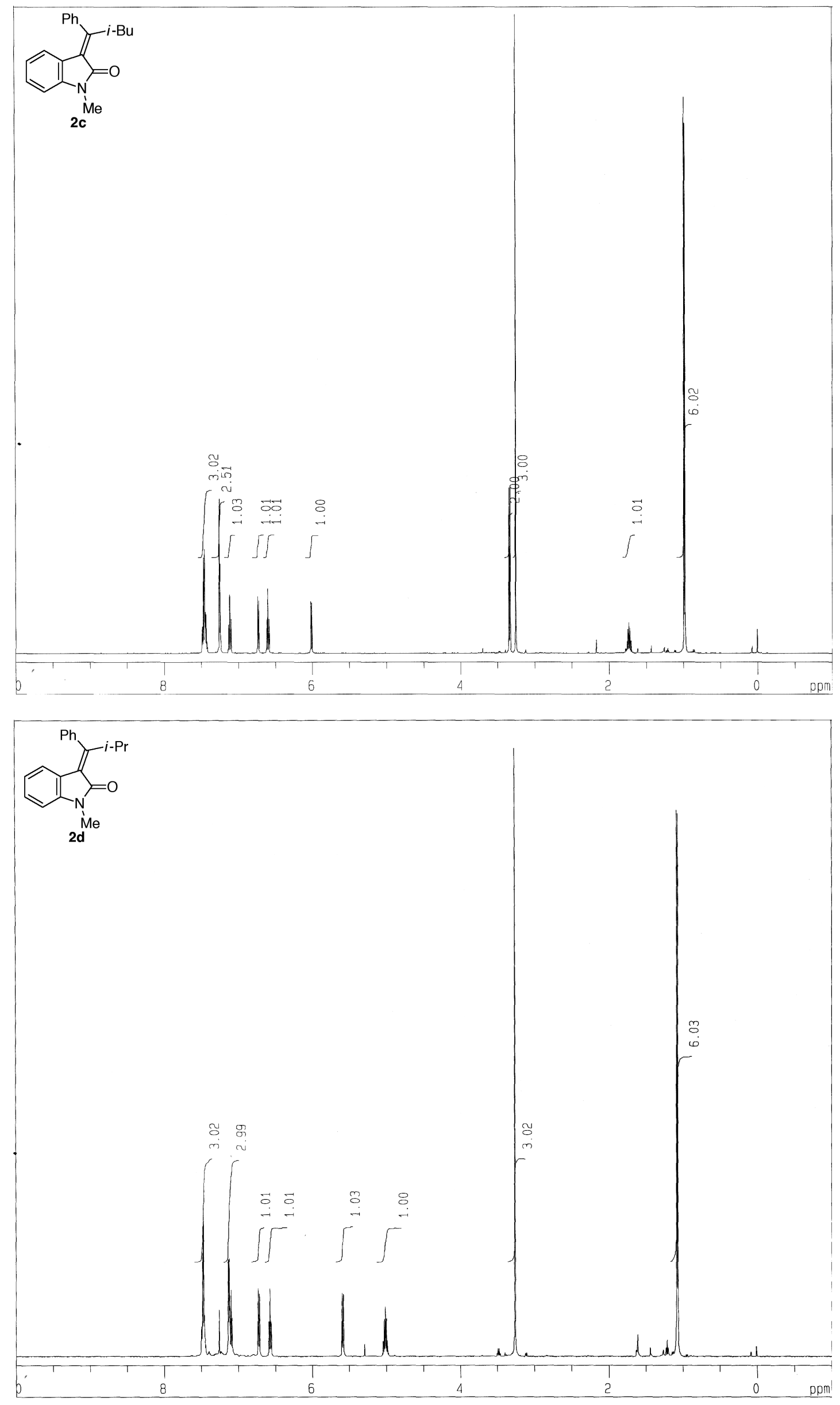

S15 

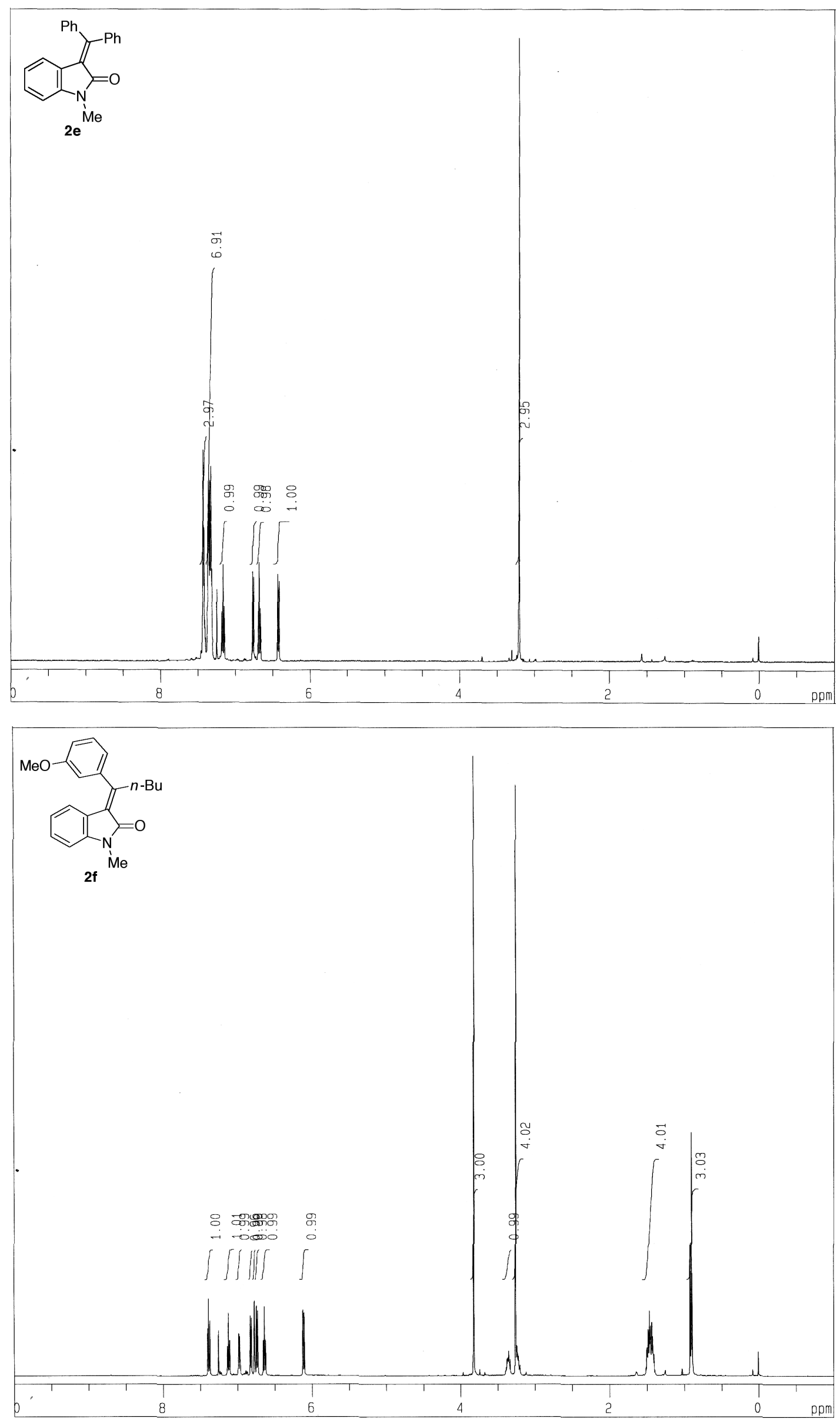

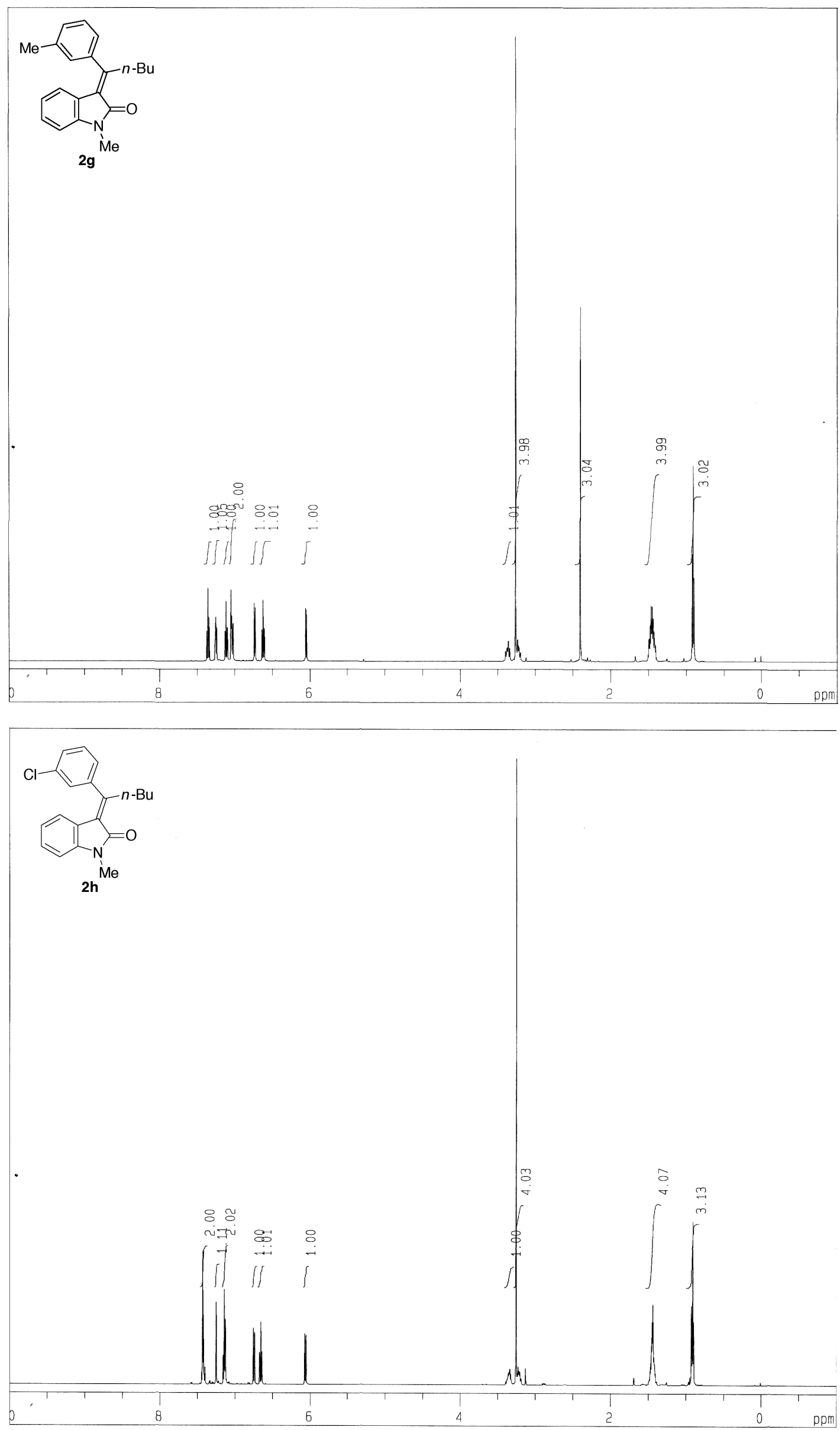

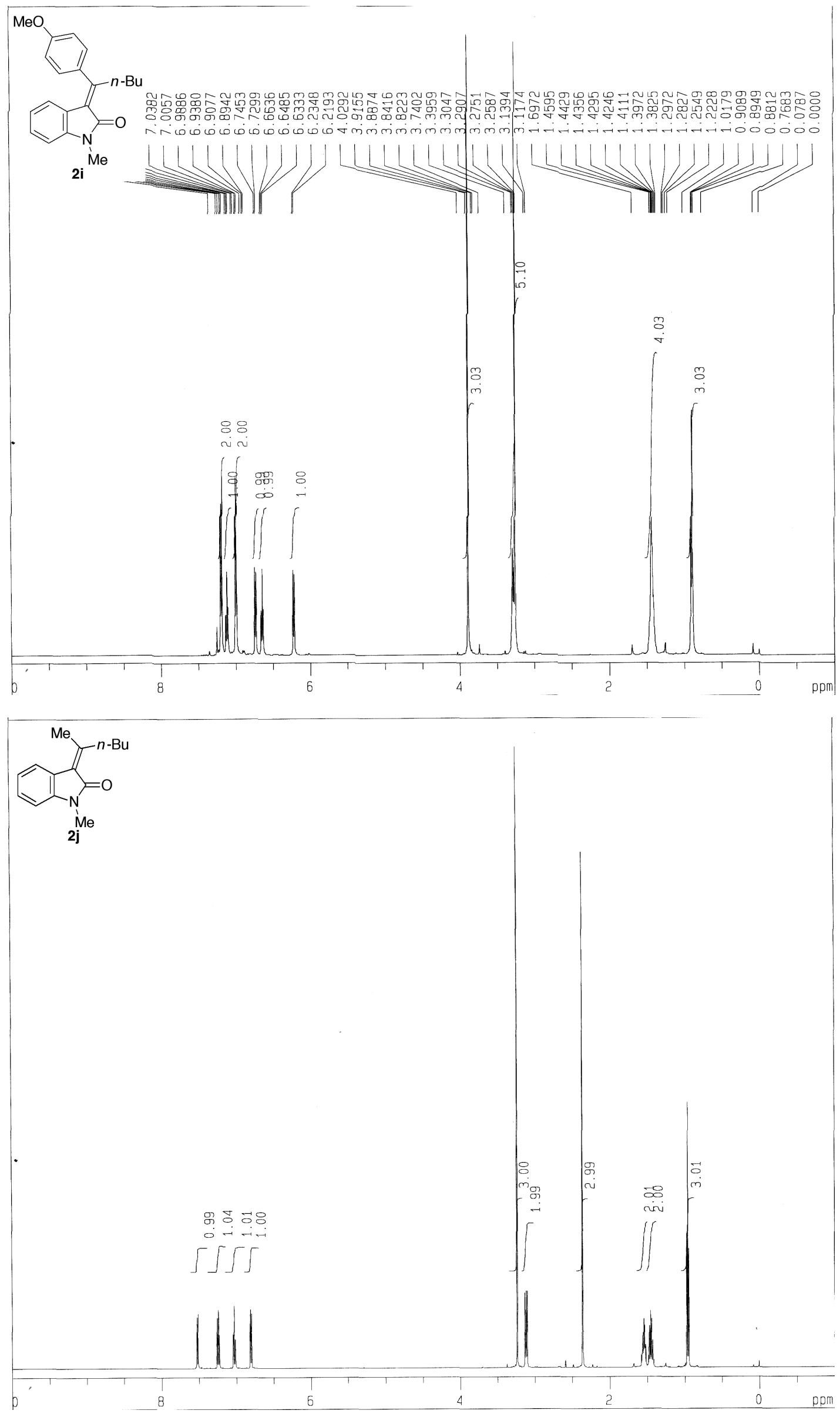

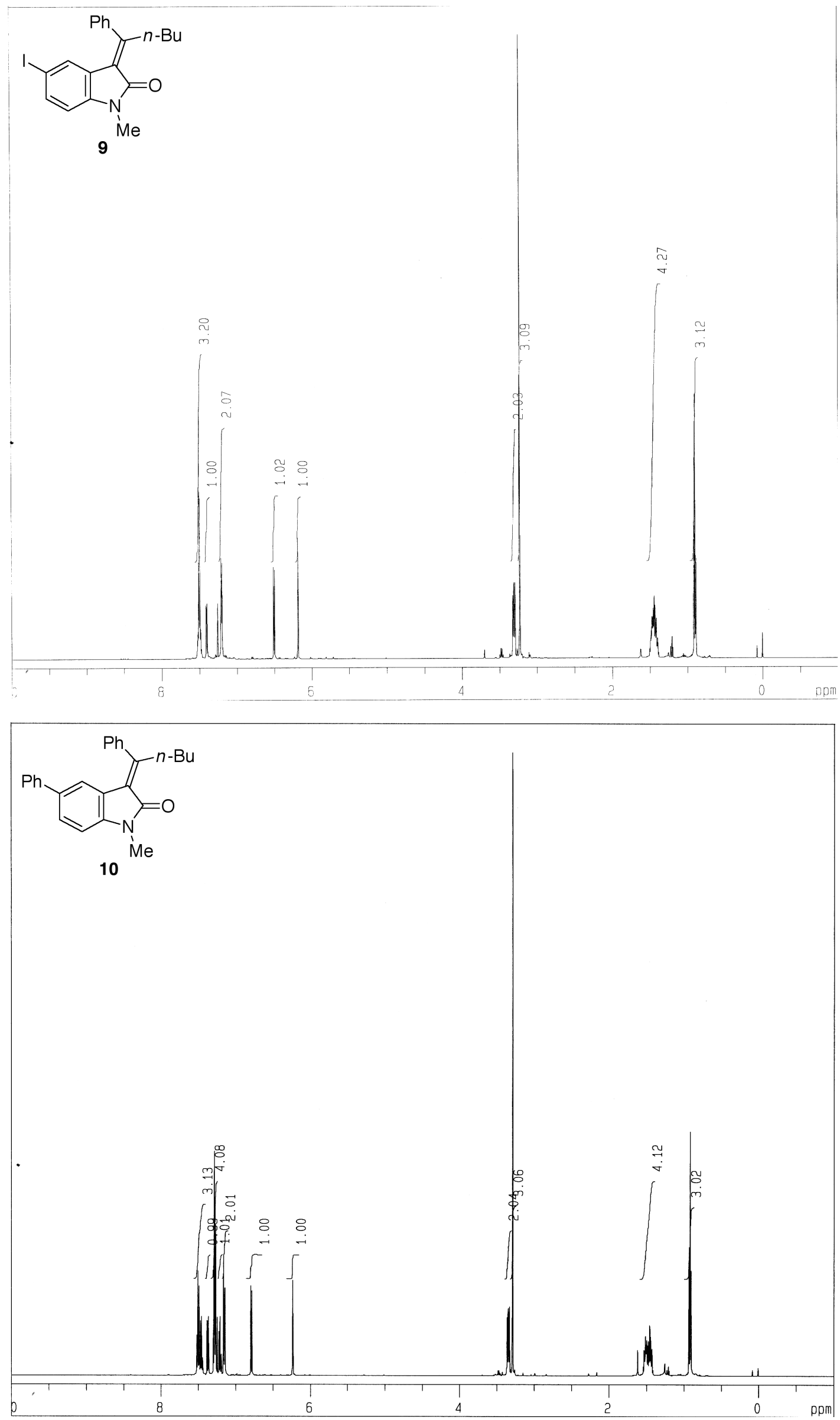

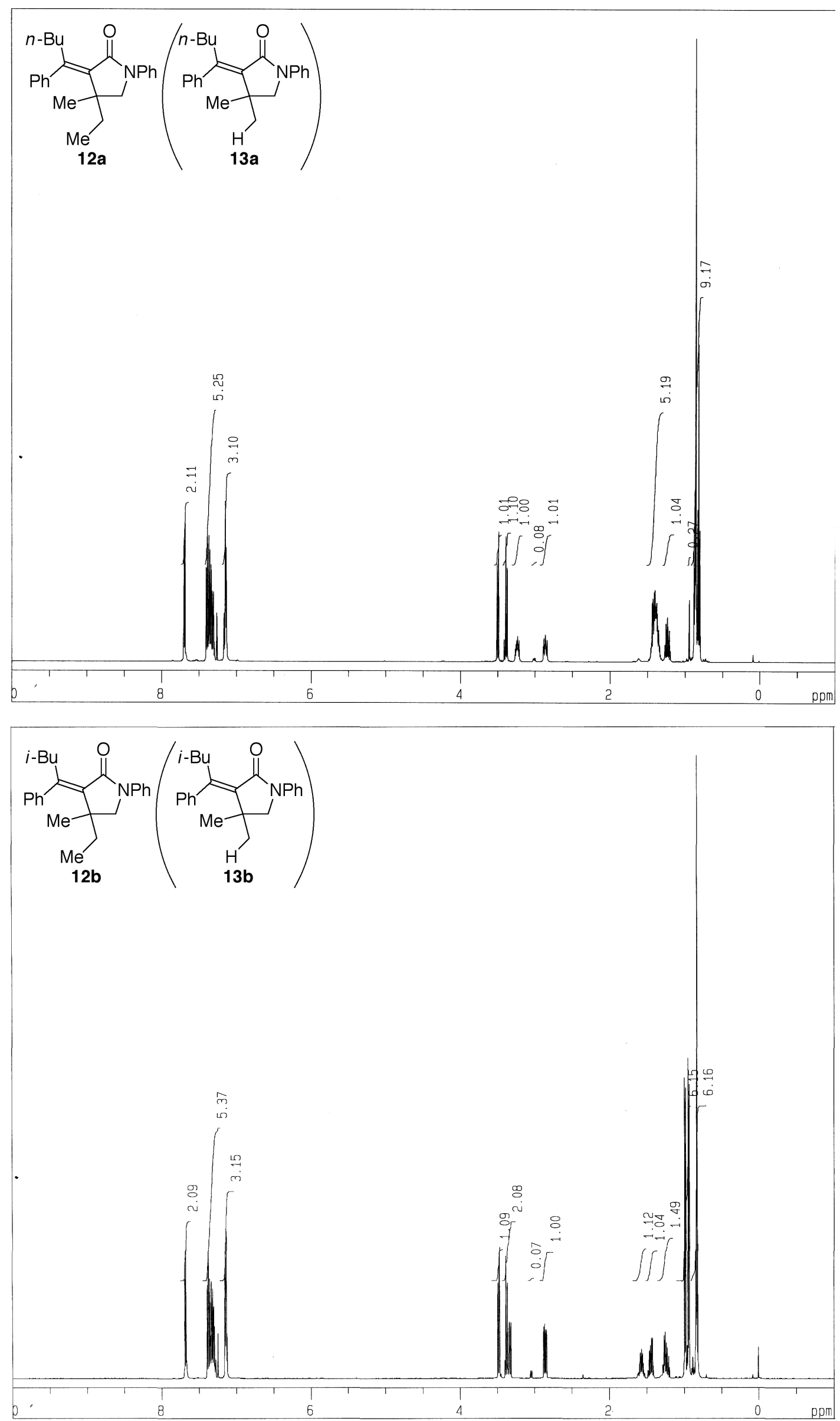